\title{
Survey of ANL Organization Plans for Word Processors, Personal Computers, Workstations, and Associated Software
}

By

Karen R. Fenske

November 1991

Computing and Telecommunications Division 


\section{ACKNOWLEDGMENTS}

The Computing and Telecommunications Division (CTD) thanks the members of the various ANL organizations who completed the informational survey forms. In most instances, these people are the ccntact persons cited on the forms. Mike Boxberger, Miriam Bretscher, Clifford Caruthers, Marydale Caruthers, John Jasunas, Fred Moszur, Debbic Mounce, Jim Regula, Rich Slade, and Samantha Vote of CTD also contributed to and revicwed the Survey.

The Survey was created with Microsoft Word on the Apple Macintosh for printing on the Apple LaserWriter. 


\section{HOW TO USE THE SURVEY}

The Computing and Telecommunications Division (CTD) has compiled this Survey of ANL Organization Plans for Word Processors, Personal Computers, Workstations, and Associated Software (ANL/TM 459, Revision 3) to provide DOE and Argonne with a record of recent growth in the acquisition and use of personal computers, microcomputcrs, and word processors at ANL. Laboratory planners, service providers, and people involved in office automation may find the Sur rey useful. It is for internal use only, and any unauthorized use is prohibited.

Readers of the Survey should use it as a reference document that (1) documents the plans of each organization for office automation, (2) identifies appropriate planners and other contact people in those organizations, and (3) encourages the sharing of this information among those people making plans for organizations and decisions about office automation. The Survey supplements information in both the ANL Statement of Site Strategy for Computing Workstations (ANL/TM 458) and the ANL Site Response for the DOE Information Technology Resources Long-Range Plan (ANL/TM 485).

CTD's procedure for compiling this Survey has been to request that each organization provide the following information in previously formatted tables:

- Contact person(s), subject, phone number, and e-mail address.

- Numbers of personal computers and workstations that existed in FY1990, that exist in FY1991 and that will exist in FY1992.

- Numbers of word processors that existed in FY 1990, that exist in FY1991, and that will exist in FY1992.

- Names and numbers of software packages that will be in use by September 1991.

- Numbers of network connections that exist in FY1991 and that will exist in FY1992.

- Additional plans for office automation.

If a cost center did not provide information for a table, we omitted that table from the Survey.

"N/A" symbolizes a number that a cost center did not provide in a completed table.

The following organizations did not participate in the FY1991 Survey:

- Argonne West--EBR-Il (Cost Center 102)

- Argonne West--Radiation Monitoring (Cost Center 551)

- Argonne West--Safety Staff (Cost Center 536)

- Argonne West--Special Materials (Cost Center 532!

- Installations (Cost Center 502)

Argonne West--Food Services (Cost Center 559) does not appear in the 1991 Survey because it has no hardwarc, software. er network connections. 
-iv- 


\section{CONTENTS}

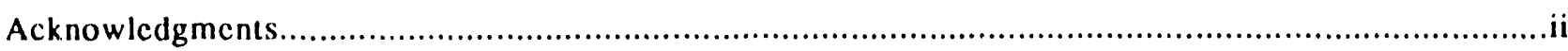

How to Use the Sur.

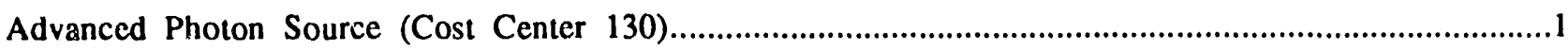

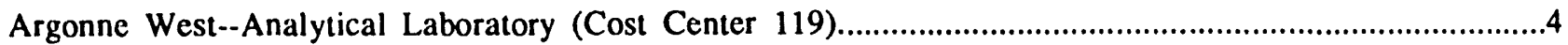

Argonne West--Computer Applications and Service (Cost Center 550).....................................................6

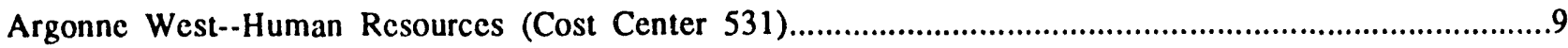

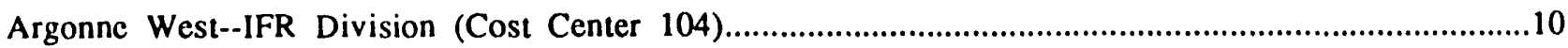

Argonne West--Information Services (Cost Center 537)..........................................................................13

Argonne West--Machine Shop (Cost Center 554),.............................................................................14

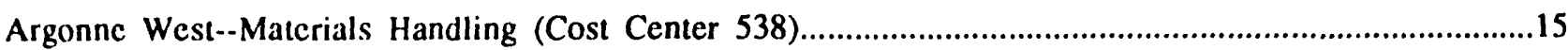

Argonne West--Office of Quality Assurance (Cost Center 561) ...................................................................17

Argonne West--Plant Services (Cost Center 557)....................................................................................19

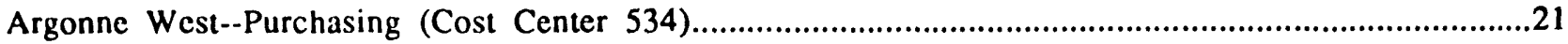

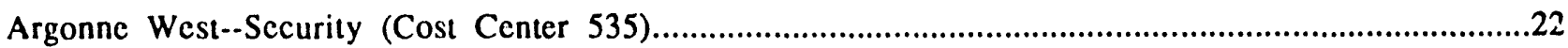

Argonne West--Site Engincering (Cost Center 556)............................................................................ 24

Argonne West--Site Manager's Office (Cost Center 530)............................................................................27

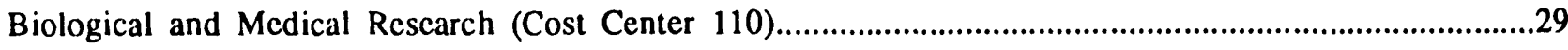

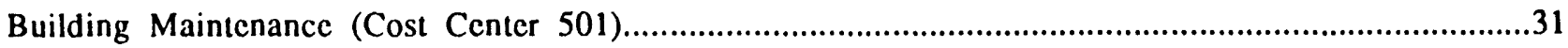

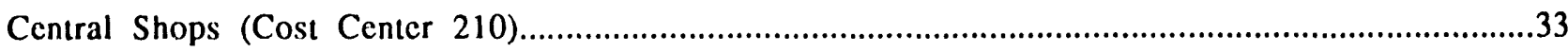

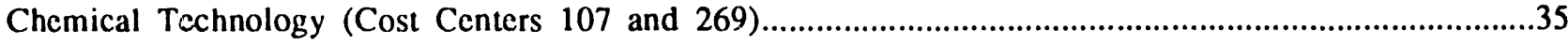

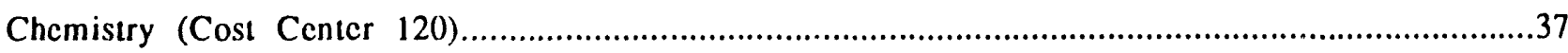

Chicf Operations Officer (Cost Center 202).....................................................................................41

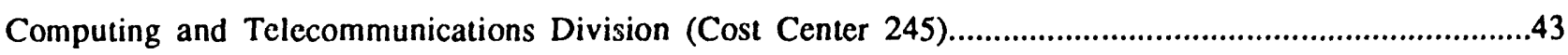

Division of Educational Programs (Cost Center 139)...............................................................................45

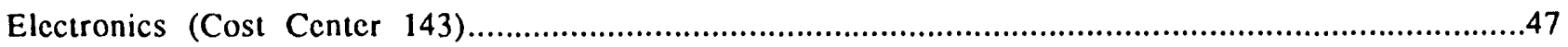

Energy, Environmental, and Biological Research Program Administration (Cost Center 274)...........................49

Encrgy, Environmental, and Biological Research Program Direction (Cost Center 174)....................................51 


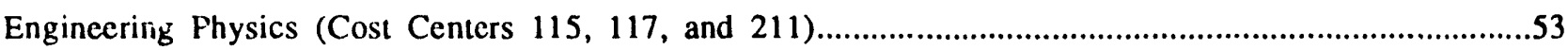

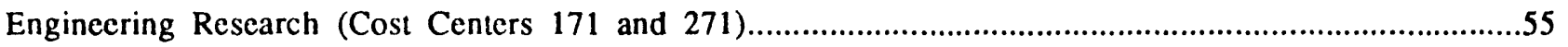

Environment, Safcty, and Hcalth/Quality Assurance Oversight (Cost Center 333)........................................57

Environment, Safety, and Health Division--Division Office (Center 235) ....................................................60

Environment, Safety, and Health Division--Dosimetry and Analytical

Services (External Dosimetry) (Cost Center 235)......................................................................................62

Environment, Safety, and Health Division--Dosimetry and Analytical

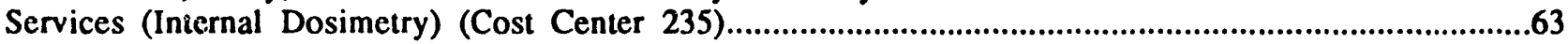

Environment, Safety, and Health Division--Environmental Protection (Cost Center 235).................................65

Environment, Safety, and Health Division--Health Physics (Cost Center 234)................................................67

Ennvironment, Safety, and Health Division--Industrial Hygiene (Cost Center 235).....................................68

Environment, Safety, and Health Division--Safety Engineering Section (Cost Center 234),................................70

Environment, Safety, and Health Division--Training and Quality Assurance (Cost Center 235)...........................72

Environmental Assessment and Information Sciences Division (Cost Center 165)...........................................73

Environmental Research Division (Cost Center 149)............................................................................76

Facilities Planning/Engineering (Cost Center 512)..................................................................................79

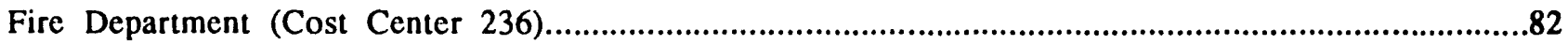

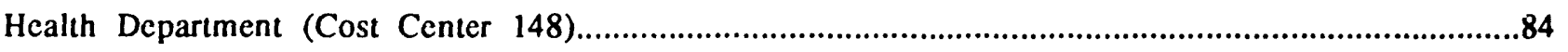

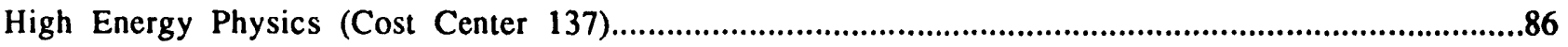

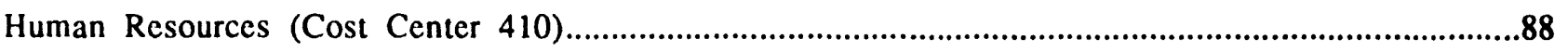

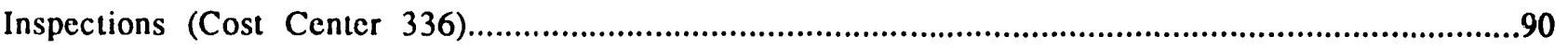

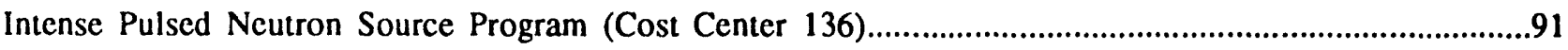

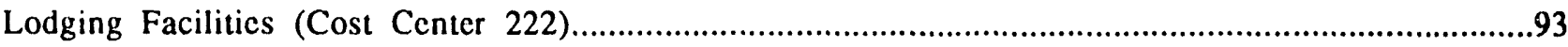

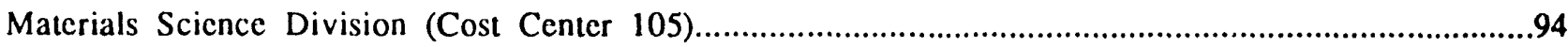

Materials and Components Technology (Cost Center 114)...................................................................96

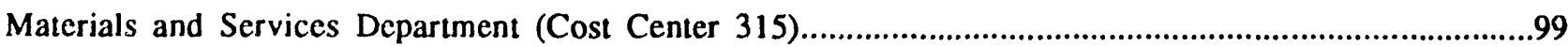

Mathematics and Computer Science (Cost Center 145) .........................................................................101

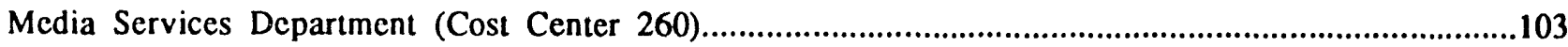

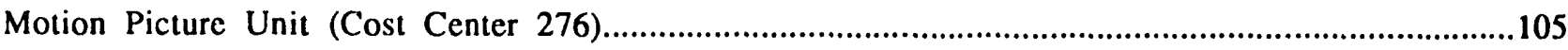

National Energy Software Center (Cost Center 246) ...........................................................................107 
Office of the Chicf Financial Officer (Cost Centers 400, 401, 402, and 403)...........................................109

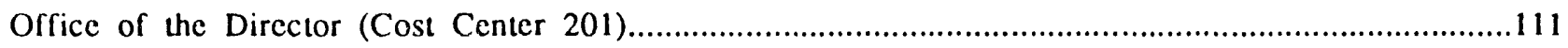

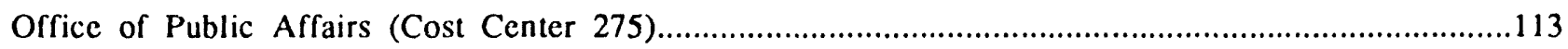

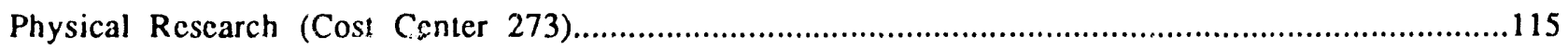

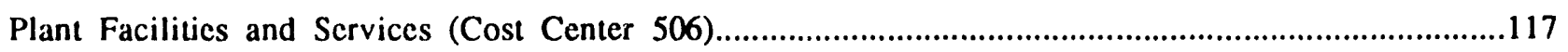

Procurement Department (Cost Center 322)....................................................................................119

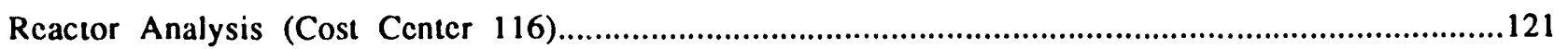

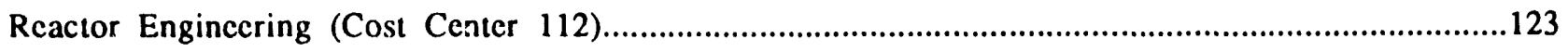

Secretarial and Clcrical Services (Cost Center 416)

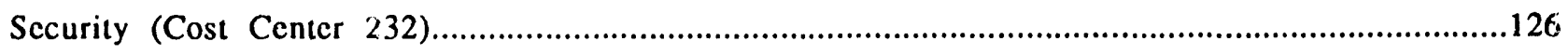

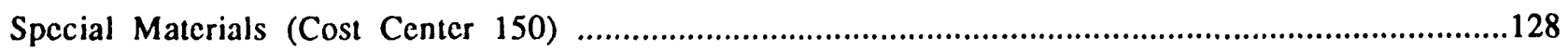

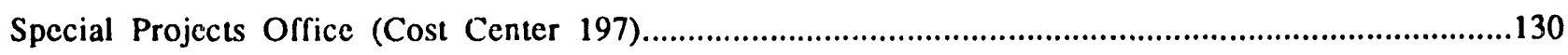

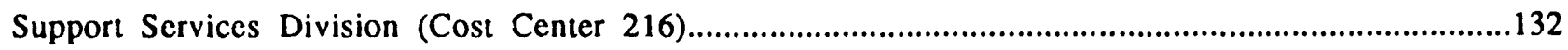

Technical Communication Services Department (Cost Center 265)..........................................................134

Technical Information Services Department (Cost Center 161)...................................................................137

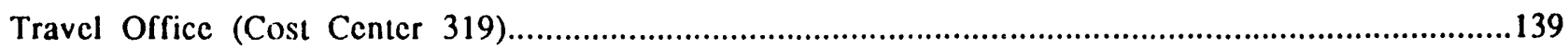

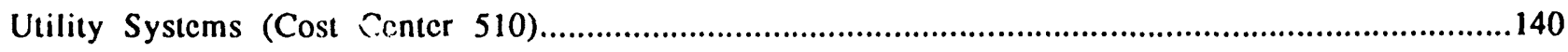

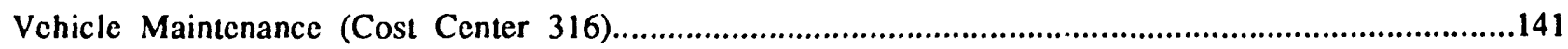

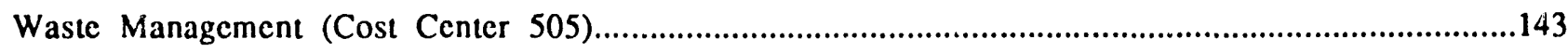


-viii- 


\section{ADVANCED PHOTON SOURCE (COST CENTER 130)}

\begin{tabular}{|llcl|}
\hline & \multicolumn{3}{c|}{ Primary } \\
& Subject & Phone & E-Mail Address \\
Contact Person & Inventory & $2-6500$ & KATORW@ANLAPS \\
Bill Kator & & & \\
\hline
\end{tabular}

\begin{tabular}{|lccc|}
\hline & Personal Computers & and & Workstations \\
& Existing & Existing & Estimated Total \\
Manufacturer & FY1990 & FY1991 & FY1992 \\
IBM (PC, AT, XT, PS/2) & 25 & 9 & 9 \\
Applc (II, Macintosh) & 15 & 64 & 64 \\
Compaq & 20 & 63 & 63 \\
Sun & 4 & 45 & 45 \\
Dec Workstation & 0 & 2 & 2 \\
VAX Station & 3 & 6 & 6 \\
AST & 0 & 2 & 2 \\
Dell & 0 & 6 & 6 \\
DTK & 0 & 4 & 4 \\
Encrex & 0 & 1 & 1 \\
Epson & 0 & 2 & 2 \\
GesPac & 0 & 1 & 1 \\
Hewlcu Packard & 1 & 1 & 1 \\
MicroStar & 0 & 1 & 1 \\
Northgatc & 0 & 1 & 1 \\
Standard & 0 & 2 & 2 \\
Toshiba & 0 & 2 & 2 \\
Zcnith & 0 & 2 & 2 \\
Zcos & 0 & 1 & 1 \\
\hline
\end{tabular}




\section{Software}

Word Processing/Editing

MacWrite

Word

WordStar

WordPerfect

Correct Grammer (1)

Utilities

Norton Commander (19)

Norton Utilities

MacTools

PC Tools

QEMM

Sidckick

Sideways

Suitcase II

After Dark

(2)

Database

DBase III

DBase IV

FileMaker

(2)

Integrated Packages Micrsoft Works MS Windows

Spreadsheet

$\begin{array}{lc}\text { Quattro } & (1) \\ \text { Lotus 1-2-3 } & \text { (5) } \\ \text { Excel } & \text { (20) }\end{array}$

Operating Systems 20/20 DOS

DOS Utility

FastBack

Communication/Terminal Emulation NCSA Telnet (4)

Kermit

Reflections

SmarTerm

VersaTerm
Graphics

MacDraw
MacPaint
MacDraft
Cricket Graph
GEM

Harvard Graphics (1)

Kaleidagraph

PaintBrush

Power Point

AutoSketch

(1)

Canvas

(1)

Network Operating Systems

$\begin{array}{ll}\text { PC NFS } & \text { (1) } \\ \text { TOPS } & \text { (3) }\end{array}$

Desktop Publishing

PageMaker

(1)

Project Management MacProject II Harvard Project Manager

Languages/Compilers

Pascal

Frortran

Turbo Basic

Turbo Lightning (1) 4th Dimension (1)

4D Compiler

C Compiler (14)

$\mathrm{C}++$ Compiler

HyperCard

CAE/CAD

AutoCAD

ORCAD

Claris CAD

Easy CAD

(1)

Math Cad 


\section{Software (continued)}

Other

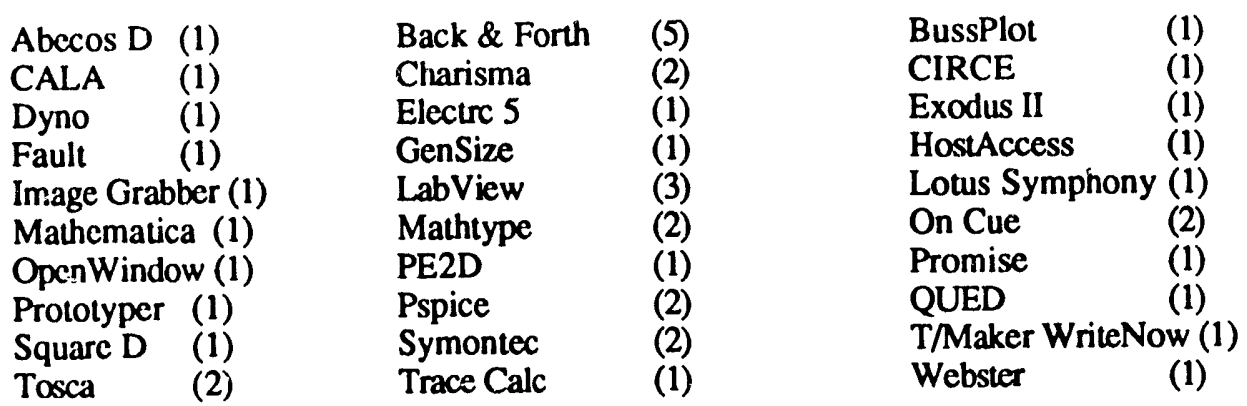

\section{Network Connections}

$\begin{array}{lc}\text { Existing } & \text { Estimated Toial } \\ \text { FY1991 } & \text { FY1992 }\end{array}$

Lab-wide Ethernet

200

200

TCP/IP

0

0

DECnet

Both

Local AppleTalk

Local 3Com

0

0

0

Planned activities and/or applications to be implemented during the next fiscal year in the areas of (1) scientific work stations, (2) personal computing, (3) local area networking, and (4) office automation:

1. Scientific Work Stations: Will probably continue to acquire scientific workstations for both UNIX and VMS environments. The stations will be replacing character cell terminals. Migration is being made towards more distributed computing.

2. Personal Computing: Will probably not continue to purchase personal computers, but rather replace them with vorkstations and $X$-terminals. Migrating toward distributed computing.

3. Loca' Area Networking: Will continue to maintain all workstations and personal computers on a local area network as part of the distributed computing environment. Will add a CISCO router to help maintain the LAN along with the wide area network.

4. Office Automation: Primary area of effort in office automation will be to implement a WYSIWYG word processor. 
ARGONNE WEST--ANALYTICAL LABORATORY (COST CENTER 119)

Primary Contacts

$\begin{array}{llcc}\text { Contact Person } & \text { Subject } & \text { Phone } & \text { E-Mail Address } \\ \text { Gordon Bemand } & \text { All } & 6-7931 & \text { N/A }\end{array}$

Personal Computers and Workstations

Manufacturer

IBM Compatibles

Apple (II, Macintosh)

Hewlett Packard 86

Compu-Add

Existing
FY1990
N/A
N/A
N/A
2

\section{Existing}

21

2

2
Estimated Tosal FY1992
23

3

2

Software

Word Processing/Editing

Word

Word Perfect

Dalabase

DBase III Plus

DBase IV

Integrated Packages

Excel

Integrator

Utility

HyperCard

Ontrack

PC Fullback

Norton Utilities

Languages

Quick Basic

Graphics

$\begin{array}{ll}\text { MacDraw II } & \text { (1) } \\ \text { MacPaint } & (1) \\ \text { Draw } & \text { (1) } \\ \text { AutoSketch } & \text { (5) }\end{array}$

Spreadsheet

Lotus 1-2-3
Quattro

Communication/Terminal Emulation

(4)

Other

Chromato Chart (1)

MS DOS

(21)

Chem Perfect

(3)

(1)

(5)
(1)

(1)

(10)

Kermit

(6) 


\section{Network Connections}

$\begin{array}{lc}\text { Existing } & \text { Estimated Total } \\ \text { FY1991 } & \text { FY1992 }\end{array}$

Lab-wide Ethernet DECnet

1

1

Planned activities and/or applications to be implemented during the next fiscal year in the areas of (1) scientific work stations, (2) personal computing. (3) local area networking, and (4) office automation:

1. Scientific Work Stations: Plan on adding computer controlled robotics in the lab hot cells.

2. Personal Computing: None planned.

3. Local Area Networking Planning on setting up a LIM system.

4. Office A tomation: None planned. 


\section{ARGONNE WEST--COMPUTER APPLICATIONS AND SERVICE (COST CENTER 550)}

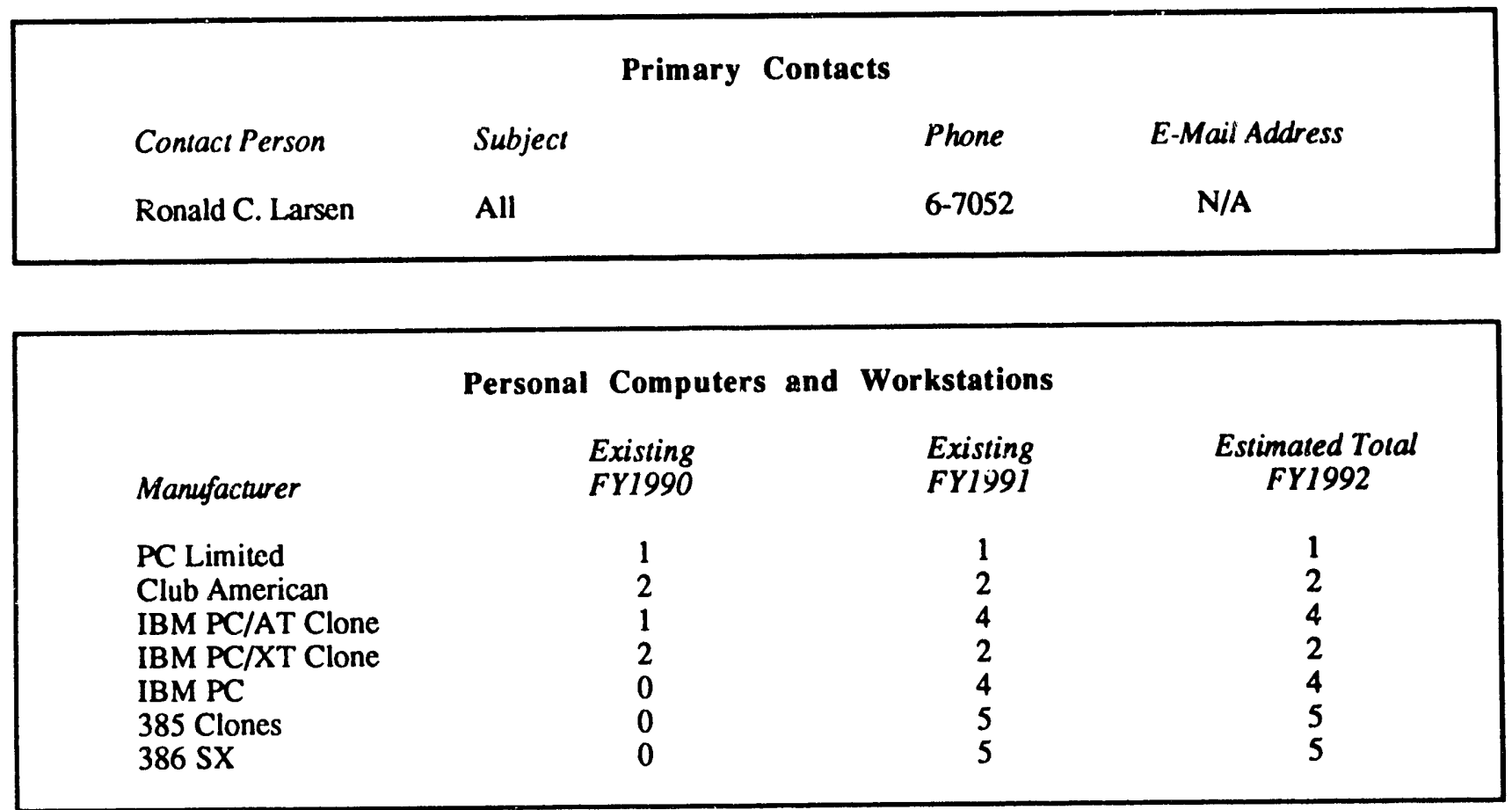

\begin{tabular}{|lccc|}
\hline & Word Processors & & \\
& Existing & Existing & Estimated Total \\
Manufacturer & FY1990 & FY1991 & \\
NBI 64 & 1 & 1 & 1 \\
NBI 5000S & 1 & 0 & N/A \\
NBI 4000S & 2 & 1 & 1 \\
NBI 2000 & 2 & 2 & 2 \\
& & & \\
\hline
\end{tabular}




\section{Software}

Word Processing/Editing

Mass-11

WordPerfoci

WordPcrfort Office (2)

$\begin{array}{ll}\text { DBase III Plus } & (5) \\ \text { DBase IV } & (7) \\ \text { FoxPro } 2.0 & (1)\end{array}$

Spreadsheet

Lotus 1-2-3

(2)

Quaturo Pro

Excel 3.0

Operating Systems

Windows 3.0

Communication/Terminal Emulation

Kermit

CrossTalk

(1)

Reflections 7

(3)

CAE/CAD

AutoCAD

(3)

\section{Languages/Compilers \\ Latice C \\ Assemble \\ Microsoft C \\ Microsoft Basic 7.0 (1) \\ Microsoft Windows (1)}

Utility

Norton Utilities (3)

Norton Backup

\section{Network Connections}

\section{Existing}

FY1991

\section{Estimated Total} FY1992

Lab-wide Ethernet

$$
\text { TCP/IP }
$$

3

Novell

20 (network)

4
3
28 (2 networks)


Planned activities and/or applications to be implemented during the next fiscal year in the areas of $(1)$ scientific work stations, (2) personal computi !, (3) local area networking, and (4) office automation:

I. Scientific Work Stations: None planned.

2. Personal Computing: IBM-PC Clones based on 80386 implementation.

3. Local Area Networking: Connection of all departmental personal computers in local area networks to share printers and other resources to facilitate mail and backup of files.

4. Office Automation: Uniform implementation of WordPerfect for word processing applications in a network environment with DBase III+ and IV for database applications. 


\section{ARGONNE WEST--HUMAN RESOURCES (COST CENTER 531)}

\section{Primary Contacts}

Contact Person

Subject

Phone

E-Mail Address

Dawn Davidson

Personal Computers

6-7323

N/A

\section{Personal Computers and Workstations}

Manufacturer

IBM (PC, AT, XT, PS/2)

Club Amcrican

Existing
FY1990
1
1

Existing
FY1991
1
1

\section{Existing}

1
Estimated Total FY1992

\section{Software}

Word Processing/Editing Mass-11

(1)
Database

DBase III Plus
(1)

Communication/Terminal Emulation

$\begin{array}{ll}\text { VTerm } & (1) \\ \text { Kermit } & (1) \\ \text { Apollo } & (1)\end{array}$

Planned activities and/or applications to be implemented during the next fiscal year in the areas of (1) scientific work stations, (2) personal computing. (3) local area networking, and (4) office automation:

1. Scientific Work Stations: None planned.

2. Personal Computing: None planned.

3. Local Area Networking: None planned.

4. Office Automation: None planned. 
ARGONNE WEST--IFR DIVISION (COST CENTER 104)

\section{Primary Contacts}

Contact Person

Colin Groves

Eric Dean

Mike Hitz

Jeff Staffon

Bill Booty/

Ed Laird
Subject

ANL-W Computer

Program Representative

IFR Division PC issues

IFR Division Apple/Mac 6-7140 issues

Engineering Dept.

Workstations Network

SUN Worksiations

EBR-II DAS

\section{Phone}

6-7246

6-7138

6-7037

6-7271
E-Mail Address

N/A

N/A

N/A

N/A

N/A

\section{Personal Computers and Workstations}

Manufacturer

IBM PC, XT, AT

MiS 80386-25

MiS 386SX

Apple MacII

Club 286

ACT $386 S X$

IBM PS $/ 2$ Model 50

IBM PS/ 2 Model 70

Altec 286

PC Limited 286

Gatcway 2000386

Altec 386

Benuly 286

Toshiba 286 Laptop

SUN $3 / 60$

HP 6400

Epson Equity 286 Laptop

\section{Existing \\ FY1990}

0

0

0

0

0

0

0

0

0

0

0

0

0

0

0

0

Existing
FY1991
29
1
7
15
36
5
19
5
4
2
4
3
1
1
3
1
1

Estimated Total FY1992

29

4

11

15

36

5

19

\section{5}

4

2

4

3

1

1

3

1

\section{Word Processors}

Manufacturer

NBI

IBM EWP 50

\section{Existing \\ FY1990}

1

0
Existing
FY1991

1

1
Estimated Total FY 1992

\section{1}




\section{Software}

Word Processing/Editing

WordPerfect

MicroSoft Word (12)

WordStar

(2)

WordVision

(2)

Spreadsheet

MicroSoft Excel

(9)

Quattro Pro
Integrased Productivity

Framework III

(25)

MicroSoft Works

(2)

MASS 11

Languages/Copmpilers

$\mathrm{C}$

$\mathrm{C}++$

Pascal

Prolog

Fortran

Database

DBase III+ (10)

DBase IV (1)

Paradox

(4)

PC Tools

(9)

RapidFilc

Filcmaker Pro

PC Plot

(19)

Paradox Engine

CAD/Graphics

Autosketch

Autoocad

DrawPcrfoct

(2)

HyperCard

Special Operating Systems

MicroSoft Windows (15)

OS2

MacDraw

Concurrrent DOS XM (3) 
Planned activities andlor applications to be implemented during the next fiscal year in the areas of (1) scientific work stations, (2) personal computing, (3) local area networking, and (4) office automation:

1. Scientific Work Stations: Integrate a MasComp Workstation with the Engineering Dept. Workstation Network (EDWN).

2. Personal Computing: Continue to upgrade PC workstations to 80386-based machine running MicroSoft Windows as older machines are replaced.

3. Local Area Networking: Shift IFR Division workload away from the ANL-W VAX and toward the EDWN PC Network.

4. Office Automation: Connect all Engineering Dept. PCs to the EDWN PC LAN, transfer existiing databases from the ANL-W VAX MASS11 to Paradox on the PC LAN, and expand database applications to include engincering project planning and tracking. 


\section{ARGONNE WEST--INFORMATION SERVICES (COST CENTER 537)}

\section{Primary Contacts}

Contact Person

Judy Krieger
Subject

All
Phone

6-7237
E-Mail Address

N/A

\section{Personal Computers and Workstations}

Manufacturer

IBM (PC, AT, XT, PS/2)

\section{Existing}

FY1990

2

\section{Existing \\ FY1991}

4

5

\section{Software}

Word Processing/Editing WordPcrfect

(1)

Spreadsheet

Lotus 1-2-3

(1)
Database

DBase III

Passport

(1)

(1)
Estimated Total FY1992

\section{Communication/Terminal Emulation}

Kermit

Utility

QFiler (1)

Planned activities and/or applications to be implemented during the next fiscal year in the areas of (1) scientific work stations, (2) personal computing. (3) local area networking, and (4) office automation:

1. Scientific Work Stations: One new station will provide C-D ROM standards from ANSI, ASME, IEEE, and UL. The other will be used for searching data bases such as Science and Technology, NTIS, Science Citation, Chemical Abstracts, etc. on C-D ROM.

2. Personal Computing: Implement graphic programs for use in newsletter.

3. Local Area Networking: None planned.

4. Office Automation: Develop experience with DBase, OCLC Passport, WordPerfect, and the new Dialog dusa bases. 


\section{ARGONNE WEST--MACHINE SHOP (COST CENTER 554)}

\section{Primary Contacts}

Contact Person

Subject

Phone

E-Mail Address

Larry Spencer

All

6-7411

N/A

\section{Personal Computers and Workstations}

$\begin{array}{ll}\text { Existing } & \text { Existing } \\ \text { FY1990 } & \text { FY1991 }\end{array}$

Estimated Total

Manufacturer

N/A

N/A

FY1992

Micro-Term

MIS 3865X-16 Computer

N/A

1

N/A

1

\section{Word Processors}

$\begin{array}{lccc}\text { Manufacturer } & \begin{array}{c}\text { Existing } \\ \text { FY1990 }\end{array} & \begin{array}{c}\text { Existing } \\ \text { FY1991 }\end{array} & \begin{array}{c}\text { Estimated Tolal } \\ \text { FY } 1992\end{array} \\ \text { Xcrox Model 6040 } & \text { N/A } & 1 & 1\end{array}$

Planned activities and/or applications to be implemented during the nexi fiscal year in the areas of (1) scientific work stations, (2) personal computing. (3) local area networking, and (4) office automation:

1. Scientific Work Stations: None planned

2. Personal Computing: None planned

3. Local Area Networking: DBase

4. Office Automation: None planned 


\section{ARGONNE WEST--MATERIALS HANDLING (COST CENTER 538)}

\section{Primary Contacts}

Contact Person

Subject

Phone

E-Mail Address

J.S. Molen

All

$6-7484$

N/A

Personal Computers and Workstations

Manufacturer

\section{Existing}

FY1990

Altec-286

H.P. Laser Jet III
0

0

\section{Existing \\ FY1991}

3

1
Estimated Total FY1992

\section{Word Processors}

Manufacturer

\section{Existing}

FY1990

\section{Existing}

FY1991

\section{Estimated Total \\ FY 1992}

Xerox

1

1

0

\section{Software}

Word Processing/Editing

WordPerfoct

Dos 5

Other

List and Mail Plus

Formfiller

49 CFR Reg Scan

29 CFR Reg Scan
(1)

(1)

(1)

(1)

(1)

(1)
Database

DBase III Plus (1)

DBase IV 


\section{Other}

$\begin{array}{lccc}\text { Manufacturer } & \begin{array}{c}\text { Existing } \\ \text { FY1990 }\end{array} & \begin{array}{c}\text { Existing } \\ \text { FY1991 }\end{array} & \begin{array}{c}\text { Estimated Total } \\ \text { FY1992 }\end{array} \\ \text { H.P. Laser Jet III } & 0 & 1 & 1\end{array}$

Planned activities anillor applications to be implemented during the next fiscal year in the areas of (1) scientific work stations. (2) personal computing, (3) local area networking, and (4) office automation:

1. Scientific Work Stations: None planned.

2. Personal Computing: Implement IBM-compatible personal computers.

3. Local Area Networking: None planned.

4. Office Automation: Upgrade from Xerox to IBM-compatible system. 


\section{ARGONNE WEST--OFFICE OF QUALITY ASSURANCE (COST CENTER 561)}

\begin{tabular}{|llcl|} 
& & Primary Contacts & \\
Contact Person & Subject & Phone & E-Mail Address \\
Ellen Christensen & All & $6-7523$ & N/A \\
\hline
\end{tabular}

\begin{tabular}{|lccc|}
\hline & Personal Computers and Workstations & \\
& Existing & Existing & Estimated Total \\
Manufacturer & FY1990 & FY1991 & FY1992 \\
IBM (PC, AT, XT, PS/2) & 1 & 1 & 3 \\
PC Limited & 2 & 2 & 2 \\
Hewkut Packard & 1 & 1 & 1 \\
ERCO & 1 & 1 & 1 \\
\hline
\end{tabular}

\section{Word Processors}

$\begin{array}{lrrr}\text { Manufacturer } & \begin{array}{c}\text { Existing } \\ \text { FY1990 }\end{array} & \begin{array}{c}\text { Existing } \\ \text { FY1991 }\end{array} & \begin{array}{c}\text { Estimated Total } \\ \text { FY } 1992\end{array} \\ \text { SilverRecd EX 78 } & \text { N/A } & \text { N/A } & \text { N/A }\end{array}$

\section{Software}

Database

DBase III Plus
CALPRO

Utilities

Norton Utilities (3)

\section{Word Processing}

Word Perfect

Mass 11

Excel

FastBack

ORPS
(5) Windows

(1) Kermit

(1) PageMaker

(1) Reflections

(1)
(1)

(1)

(1) 
Planned activities andlor applications to be implemented during the next fiscal year in the areas of $(I)$ scientific work stations, (2) personal computing, (3) local area networking, and (4) office automation:

1. Scientific Work Stations: None planned

2. Personal Computing: Implementing access to ORPS for Occurrencing Reporting.

3. Local Area Networking: None planned.

4. Office Automation: Institute tracking and trending of action items. 


\section{ARGONNE WEST--PLANT SERVICES (CC'ST CENTER 557)}

\section{Primary Contacts}

Contact Person

Subject

Phone

E-Mail Address

Bruce C. Gay

All

6-7715

N/A

\section{Persona! Computers and Workstations}

$\begin{array}{cccc} & \text { Existing } & \text { Existing } & \text { Estimated Total } \\ \text { Manufacturer } & \text { FY1990 } & \text { FY1991 } & \text { FY1992 }\end{array}$

Club American

APS

5
0
0
0
0

$\begin{array}{rr}5 & 5 \\ 1 & 1 \\ 8 & 12 \\ 1 & 1 \\ 1 & 1\end{array}$

\section{Word Processors}

\section{Existing}

FY1990

Existing

FY1991

Estimated Total

FY 1992

IBM Wheclwriter 6

Xerox 620

Xerox 6020

IBM Wheclwriter 30

$\begin{array}{ll}2 & 2 \\ 1 & 1 \\ 1 & 1 \\ 0 & 1\end{array}$

\section{Software}

Word Processing/Editing

Mass-11

(4)

Deskmate

WordPerfect 5.1

Database

DBase III Plus (3)

DBase IV

Deskıop Publishing

Always

Sideways

(3)
AutoSketch
(5)
DrawPerfect
(2)

Spreadsheet

Lotus 1-2-3

(6)

Quattro-Pro

Security

$\begin{array}{ll}\text { FluShot } & (1) \\ \text { PC-Vauit } & (2)\end{array}$




\section{Software (continued)}

Communication/Terminal Emulation

Kermit

(8)

Other
Utility

Norton Commander

(11)

Wonder

(6)

Mouse

$\begin{array}{llll}\text { Registrar } & \text { (1) } & \text { Fast Regs } & \text { (2) } \\ \text { Regscan } & \text { (9) } & \text { SARA! } & \text { (2) } \\ \text { TopPriority } & \text { (4) } & \text { Form Tool } & \text { (1) } \\ \text { Laddr } & \text { (1) } & \text { Whereabouts } & \text { (1) } \\ \text { MicroSoft Project (2) } & \text { Windows } & \text { (2) }\end{array}$

Norton Utilities

(1)

(1)

(2)

Planned activities and/or applications to be implemented during the next fiscal year in the areas of (1) scientific work stations, (2) personal computing, (3) local area networking, and (4) office automation:

1. Scientific Work Stations: None planned.

2. Personal Computing: Continue to develop maintenance engineering support applications utilizing existing available commercial software.

3. Local Area Networking: None planned.

4. Office Automation: Continue to develop and implement office aitomation improvements using available commercial software. 


\section{ARGONNE WEST--PURCHASING (COST CENTER 534)}

\section{Primary Contacts}

Contact Person Subject Phone E-Mail Address
G.B. Wray
All
6-7243
N/A

\section{Personal Computers and Workstations}

$\begin{array}{lccr}\text { Existing } & \text { Existing } & \text { Estimated } \\ \text { Manufacturer } & \text { FY1990 } & \text { FY1991 } & \text { FY 1992 } \\ \text { Gatcway } & 1 & 4 & 4 \\ \text { ACT } & 4 & 4 & 4 \\ \text { ALTEC } & 2 & 2 & 2\end{array}$

\section{Software}

Word Processing/Editing

Database

Mass-11

(1)

DBase III Plus

Lotus 1-2-3

Quattro Pro

(1)

(1)

(1)

Communication/Terminal Emulation

Reflections

(5)

Planned activities and/or applications to be implemented during the next fiscal year in the areas of (1) scientific work stations, (2) personal computing. (3) local area networking, and (4) office automation:

1. Scientific Work Stations: None planned.

2. Personal Computing: Four (4) additional personal computers will be procured with Word Perfect and Reflections software (to emulate AMPS) to replace aging H.P. terminals

3. Local Area Networking: Establish procurement LAN w/Novell Netware 286 Version 2.15 .

4. Office Automation: None planned. 


\section{ARGONNE WEST--SECURITY (COST CENTER 535)}

Primary Contacts

$\begin{array}{llll}\text { Contact Person } & \text { Subject } & \text { Phone } & \text { E-Mail Address } \\ \text { Manny Sanchez } & \text { All } & 6-7347 & \text { N/A }\end{array}$

\section{Personal Computers and Workstations}

Manufacturer

IBM (PC, AT, XT, PS/2)

PC Limiled

Club American

Hewleu Packard

MIS Computer

Existing
FY1990
2
3
2
1
0

Existing
FY1991
2
3
2
1
5

Estimated Total FY1992

3
3
2
1
6

\section{Software}

Word Processing/Editing

Mass-11

WordPerfoct

PC Write

Grammatik

Leading Edge

Database

DBase III Plus

File Express

QFiler

(1)

(12)

(2)

(3)

(2)

(4)

(9)

(6)

Utility

MS Windows

Norton Utilities

Onside

Tool Box

Everex Board
(2)

(3)

(2)

(2)

(1)
Graphics

Express Graphics

One Step Graphic \& Palette Plus

Surfer

Image

(5)

(1)

(1)

(1)

Spreadsheet

Express Calc

Security

G 6000 Key and Core Control

(1)

PC Lock

Assess VA

PC Vault

(1)

(1)

FTR

Superlok 300
(1)

(4) 


\section{Software (continued)}

\section{CAE/CAD}

US Metric Conversion (1)

MathCAD

(2)

Generic CAD

(1)

Other

DOS

PC Fullback

Reflections

(1)
Project Management

Softbytes

Managex

(1)

Planned activities andlor applications to be implemented during the next fiscal year in the areas of $(1)$ scientific work stations, (2) personal computing. (3) local area networking, and (4) office automation:

1. Scientific Work Stations: None planned.

2. Personal Computing: None planned.

3. Local Area Networking: None planned.

4. Office Automation: None planned. 


\section{ARGONNE WEST--SITE ENGINEERING (COST CENTER 556)}

\begin{tabular}{|llcl|}
\hline & \multicolumn{3}{c|}{ Primary Contacts } \\
Contact Person & Subject & Phone & E-Mail Address \\
& & & \\
John K. Ammon & All & $6-7505$ & N/A \\
Gene K. Sherman & All & $6-7353$ & N/A \\
Roger L. Black & All & $6-7199$ & N/A \\
\hline
\end{tabular}

\begin{tabular}{|lccc|}
\hline & Personal Computers & and & Workstations \\
& Existing & Existing & Estimated Total \\
Manufacturer & FY1990 & FY1991 & 4 \\
IBM ( AT, XT) & 4 & 4 & 6 \\
Apple (II, Mac, CX) & 4 & 6 & 1 \\
Compaq & 0 & 1 & 20 \\
Club American & 20 & 20 & 1 \\
Intergraph Interact 32c & 1 & 1 & 31 \\
A.C.T & 0 & 24 & 13 \\
Gateway & 13 & 13 & 1 \\
M.I.S. & 0 & 1 & 1 \\
Z.I.P. & 1 & 1 & 1 \\
Dauphin Laptop & 1 & 1 & 2 \\
S \& M & 0 & 2 & \\
\hline
\end{tabular}

\section{Word Processors}

$\begin{array}{lccc}\text { Existing } & \text { Existing } & \text { Estimated Total } \\ \text { Manufacturer } & \text { FY1990 } & \text { FY1991 } & \text { FY } 1992 \\ \text { SilverRced } & 1 & 1 & 1\end{array}$

\section{Software}

Word Processing/Editing

Word

MacWrite

WordPerfect 5.1

Project Management

TimeLine 4.0

Permaster Advance (2)

Surfer
Graphics

MacPaint

(1)

ChartMaster

CrickelDraw

(1)

EPS Illustrations

Paste Ease

SuperPaint

Adobe Illustrator

Impact Images

MacDraft

MacDraw

CricketPresent

CricketGraph 


\section{Software (continued)}

Database

DBasc III

DBasc III+

(2)

(4)

Spreadsheet

Framcwork III (10)

Quaturo Pro (10)

Works

Networking

Novell AFT V2.15c

Novell ELS V1.1

Utility

Norton 5.0

MAC Tools

Virex

Excel

(4)

(2)
Desktop Publishing

PageMaker

Express

(1)

(1)

Communication/Terminal Emulation

Procomm Plus (1)

DOECOMM

Kermit

Carbon Copy

(3)

CAD/CAM

Microstation 32 (1)

AutoCAD Relea' e 11 (3)

AutoCAD Relea e 10 (37)

Other

AWORKS

Coach

(1)

Grammitik 4

\section{Network Connections}

\section{Existing \\ FY1991}

Local AppleTalk

Novell LAN \# 1

Novell LAN\#2

Lab-wide Telllabs
4

37

13

4
Estimated Total FY1992

4

40

15 
Planned activities and/or applications to be implemented during the next fiscal year in the areas of (1) scientific work stations, (2) personal computing, (3) local area networking, and (4) office automation:

1. Scientific Work Stations: None planned at the present, but will continue to evaluate their value as an engineering platform and drafing tool.

2. Personal Computing: We will increase both in the quantity and use of personal computers. We will look at using one for the tracking of tasks and resources.

3. Loc: ' Area Networking: We will increase the number of users once we have upgraded to the current version of Novell and will look at bridging to other LANs.

4. Office Automation: CDOCS (Classified Document Control System) is and will be expanded and used for storage of all records. 
ARGONNE WEST--SITE MANAGER'S OFFICE (COST CENTER 530)

Primary Contacts

Contact Person

Subject

All

Phone

Jerry Ward

6-7108

E-Mail Address

N/A

\section{Personal Computers and Workstations}

$\begin{array}{cccc} & \text { Existing } & \text { Existing } & \text { Estimated Total } \\ \text { Manufacturer } & \text { FY1990 } & \text { FY1991 } & \text { FY1992 }\end{array}$

PC Limited

ACT 386

ALTECH

MacIntosh

1
0
0
0

1
3
2
4

1

4

2

\section{Word Processors}

$\begin{array}{lccc} & \text { Existing } & \text { Existing } & \text { Estimated Total } \\ \text { Manufacturer } & \text { FY1990 } & \text { FY1991 } & \text { FY } \\ \text { NBI } & 1 & 0 & 0 \\ \text { Xerox } & 1 & 1 & 0\end{array}$

\section{Software}

Word Processing/Editing

Word

Mass-11

WordPerfoct

Communication/Terminal Emulation

Kermit (4)
(1)

(5)

Utility

SystemMate II (1)

Norton Backup (2)

Excel

(4)

Screenblanker (3)

Database

FoxPro

DBase iV

Relational Report Writer (1)

Windows

MS Project for Windows (1) 
Planned activities andlor applications to be implemented during the next fiscal year in the areas of (1) scientific work stations, (2) personal computing. (3) local area networking, and (4) office automation:

1. Scientific Work Stations: None planned.

2. Personal Computing: One MacIntosh II; One IBM PC Clone

3. Local Area Networking: None planned.

4. Office Automation: None planned. 


\section{BIOLOGICAL AND MEDICAL RESEARCH (COST CENTER 110)}

\begin{tabular}{|llll|}
\hline & & Primary & Contacts \\
Contact Person & Subject & Phone & E-Mail Address \\
Susan Bart & NBI & $2-3821$ & N/A \\
Ken Groh & ADAC & $2-3861$ & GROH@ANLBEM \\
Carol Fox & Inventory & $2-3945$ & FOX@ANBIOR \\
\hline
\end{tabular}

\section{Personal Computers and Workstations}

Manufacturer

IBM (PC, AT, XT, PS/2)

Apple (II, Macintosh)

ADAC

MICROLAB

DIGITAL

AST Rescarch

Hewlctt Packard

Waters Assoc

DTK 386SX

3Com 401S

Microstar 286

Microstar 386

Compaq

DIGITAL MicrovaxII

DIGITAL 3100 Morbel 76

Existing
FY1990
5
6
5
1
5
4
3
1
0
0
0
0
0
0
0

\section{Existing
FY1991}

FY1991

20
7
1
0
6
7
4
0
6
1
1
1
2
2
1

Estimated Total FY1992

23
7
1
0
7
7
4
0
8
1
2
1
3
2
2

\section{Word Processors}

$\begin{array}{lccc} & \text { Existing } & \text { Existing } & \text { Estimated Total } \\ \text { Manufacturer } & \text { FY1990 } & \text { FY1991 } & \text { FY } 1992 \\ \text { IBM } & 3 & 5 & 5 \\ \text { NBI } & 12 & 0 & 0\end{array}$

\section{Software}

Word Processing/Editing

Text Editor

WordPerfect

Languages/Compilers

BASIC

FORTRAN
Spreadsheet

Lotus 1-2-3

(2)

Utility

Textra

(1)

DNASTAR 


\section{Network Connections}

Existing

FY1991

Lab-wide Ethernet

DECnet

TCP/IP
30

9
Estimated Total FY19?2

31

11

Planned activities and/or applications to be implemented during the next fiscal year in the areas of (1) scientific work stations, (2) personal computing, (3) local area networking, and (4) office automation:

1. Scientific Work Stations: None planned.

2. Personal Computing: None planned.

3. Local Area Networking: Connect specific PCs to VAX systems for data transfer back and forth.

4. Office Automation: Connect secretarial PCs to each other using PCSA networking system. 


\section{BUILDING MAINTENANCE (COST CENTER 501)}

\begin{tabular}{|llcl|}
\hline & \multicolumn{3}{c|}{ Primary } \\
& Subject & Phontacts & E-Mail Address \\
Contact Person & Workstations & $2-5992$ & N/A \\
Lillian Brockman & Manager & $2-5241$ & N/A \\
Jim Huggins & & & \\
\hline
\end{tabular}

\section{Personal Computers and Workstations}

Manufacturer

IBM (PC, AT, XT, PS/2)
Existing
FY1990

9
Existing

FY1991

12
Esttimated Total FY1992

20

\section{Word Processors}

Manufacturer

NBI

\section{Existing \\ FY1990}

2
Existing

FY1991

2

\section{Estimated Tolal} FY 1992

0

\section{Software}

Word Processing/Editing

Word

(2)

Word Perfect

(3)

Graphics

Dalabase

DBase III Plus
DBase IV

(1)

(1) 
Planned activities and/or applications to be implemented dwring the next fiscal year in the areas of (1) sciensific work stations, (2) personal computing. (3) local area networking, and (4) office automation:

1. Scientific Work Stations: None planned.

2. Personal Computing: None planned.

3. Local Area Networking: None planned.

4. Office Automation: None planned. 


\section{CENTRAL SHOPS (COST CENTER 210)}

\section{Primary Contacts}

Contact Person

Subject

Phone

E-Mail Address

Al Schneider

All

$2-7082$

N/A

\section{Personal Computers and Workstations}

Manufacturer

IBM (PC, AT, XT, PS/2)

Compaq

Gateway

Texas Instruments

$\begin{array}{ll}\text { Existing } & \text { Existing } \\ \text { FY1990 } & \text { FY1991 }\end{array}$

12

2

0

0
Estimated Total FY1992

\section{Software}

Word Processing/Editing

Word

(12)

Database

DBaselV

Mig. Sirop Cuntrol

Spreadsheet

Multiplan

(3)
Graphics

CHART

(2)

Communication/Terminal Emulation

Kermit

A

(7)

MSE-Estimating (7)

Network Connections

\section{Existing}

FY1991

Local 3Com

Novell
0

\section{Estimated To!al FY1992}

AutoCAD

SmartCAM 
Planned activities and/or applications to timplemented during the next fiscal year in the areas of (1) scientific work stations. (2) personal computing, (3) local area networking, and (4) office automation:

1. Scientific Work Stations: None planned.

2. Personal Computing: Existing programs.

3. Local Area Networking: Novell network for Ceritral Shops office and shop areas. Shop control software and bar code data acquisition.

4. Office Automation: Order entry, job tracking, shop control, inventory control, purchasing/receiving, data acquisiition. 


\section{CHEMICAL TECHNOLOGY (COST CENTERS107 AND 269)}

\section{Primary Contacts}

\begin{tabular}{llll} 
Contact Person & Subject & Phone & E-Mail Address \\
Stcphen Gabclnick & $\begin{array}{l}\text { Pcrsonal Computers } \\
\text { Workstations } \\
\text { Inventory }\end{array}$ & $2-4365$ & B17146 AT ANLCMT \\
\hline
\end{tabular}

\section{Personal Computers and Workstations}

Manufacturer

IBM (PC, AT. XT, PS/2)

Apple (II, Macintosh)

Compaq

Zenith

AST Rescarch

AMT

Toshiba

Hewleul Packard

DEC PC350

DEC VAXstations

Galcway 2000

\section{Existing \\ FY1990}

27

25

2

1

31

2

1

3

5

5

0

\section{Existing \\ FY1991}

30

35

5

1

36

0

1

4

5

10

12

\section{Estimated Total} FY 1992

30

40

6

1

36

0

2

4

5

12

25

\section{Software}

Word Processing/Editing

Word

KEDIT

Mass-11

WordPerfect

Database

DBasc III Plus

Project Management

TimeLine

Languages/Compilers

Turbo Pascal

FORTRAN

Basic
(1)

(50)
Graphics

MacDraw II

SuperChart

SuperImage

Mass-11 Draw

Cricket Graph

Corel Draw

Desktop Publishing

IBM Interleaf

Expressionist

Integrated Packages

Excel

Communication/Terminal Emulation

VTerm (20)

SmarTerm 240 (3)

Reflection

(10)

Kermit 
Software (continued)

Utility

Norton Utilities (10)

PC Tools

Diskfit

Other

Desquiew

CAE/CAD

AutoCAD

(3)

\section{Network Connections}

\section{Existing}

FY1991

60

60

DECnet

Both

Lab-wide AppleTalk
30

\section{Estimated Total FY1992}

80

80

40

Planned activities andlor applications to be implemented during the next fiscal year in the areas of (1) scientific work stations, (2) personal computing. (3) local area networking, and (4) office automation:

1. Scientific Work Stations: IBM RISC/6000 for computational chemistry.

2. Personal Computing: Continued purchase of $P C$ clones and MacIntosheds for office automation:

3. Local Area Networking: Installation of twisted-pair (10 Base-T) Ethernet web in addition to existing thin wire and thick wire. Installation of CISCO router/bridge.

4. Office Automation: See above. 


\section{CHEMISTRY (COST CENTER 120)}

\section{Primary Contacts}

$\begin{array}{llll}\text { Contact Person } & \text { Subject } & \text { Phone } & \text { E-Mail Address } \\ \text { Ron Van Buskirk } & \text { All } & 2-3684 & \text { VANBUSKIRK@ANLCHM }\end{array}$

\section{Personal Computers and Workstations}

Manufacturer

IBM (PC, AT, XT, PS/2)

Apple (II, Macintosh)

Compaq

Sun

Zenith

PC Limited

Standard Prod.

AST Rescarch

Dell System

Microstar

Digital

DataGeneral

Nicolet

Hewlett Packard

Haxcline

Lundy

IBM PS/6000

Toshiba

Gatcway

LOGOS ACT

\section{Existing}

FY1990

64

111

1

1

1

2

8

2

5

1

4

1

1

1

1

1

0

1

5

\section{Existing \\ FY1991}

\section{5}

144

1

3

1

1

8

2

8

1

8

1

1

1

1

1

1

1

5
Estimated Total FY1992

55
150
1
3
1
2
8
2
8
1
9
1
1
1
1
1
3
1
8
1

\section{Word Processors}

Manufacturer

IBM
Existing
FY1990

5
Existing

FY1991

4
Estimated Total FY 1992 


\section{Software}

Languages/Compilers

IBM Basic

IBM Fortran

(2)

LS C

LS Pascal

MacBasic

MS Quick Basic

MS C

(4)

MS Quick C

QuickBasic

MacFortran

Turbo Pascal

(2)

(5)

(1)

Word Processing/Editing

Chem Text

WordPerfect

WordStar

WordUp

Endnote

PC Write

MS Word

(4)

Database

DBase IV

WingZ

Double Helix

Foxbase

(2)

Spreadsheet

$$
\begin{aligned}
& \text { Lotus 1-2-3 } \\
& \text { PC Calc } \\
& \text { Excel }
\end{aligned}
$$

Integrated Packages

SAS

Utility

Norton Advanced Utilities

Norton Commander

Sidckick

PC Tools

Mac Tools
Graphics

$\begin{array}{ll}\text { ANSI Graph } & (1) \\ \text { Charting Gallery } & (1) \\ \text { ChemDraw } & (1) \\ \text { CricketDraw } & (2) \\ \text { CricketGraph } & (7) \\ \text { ExpressGraph } & (1) \\ \text { Framework II } & (1) \\ \text { HP Drawgal } & (1) \\ \text { Kaleidagraph } & (4) \\ \text { MacDraft } & (6) \\ \text { MacPlot } & (1) \\ \text { PC Plot } & (3) \\ \text { TechGraph PD } & (2)\end{array}$

Communication/Terminal Emulation

$\begin{array}{ll}\text { Emutek } & (1) \\ \text { MacTerminal } & (1) \\ \text { PC/Intercomm } & (1) \\ \text { PC Talk } & (1) \\ \text { SmartTerm } & \text { (2) } \\ \text { Versaterm } & \text { (3) }\end{array}$

Desktop Publishing

Ready Set Go

(5)

Project Management

Harvard Project Manager (1)

SuperProject

(1)

Mac Project

(1)

\section{CAE/CAD}

McCad SCM-1 (1)

McCad SMT-1 (1)

Generic CAD 


\section{Software (continued)}

Other

$\begin{array}{lrll}\text { Alchemy } & (1) & \text { MS Macro ASM } & (1) \\ \text { Disk Optimizer } & (1) & \text { MS Windows } & (3) \\ \text { EG\&G Ace } & (2) & \text { MS Works } & (1) \\ \text { Expressionist } & (1) & \text { Natl. Inst. Dev. Pkg. } & (6) \\ \text { FastBack } & (1) & \text { NB488 } & (1) \\ \text { First Aid } & (1) & \text { Passage } & (1) \\ \text { Halo } & (3) & \text { PC Model } & (1) \\ \text { Higgins } & (1) & \text { ProFile } & (1) \\ \text { HP UV/VIS } & (1) & \text { Q\&A } & (1) \\ \text { IGOR } & (3) & \text { Readability } & (1) \\ \text { MacEQN } & (1) & \text { Reflex Plus } & (2) \\ \text { MacRabbit } & (2) & \text { Semantic } & (1) \\ \text { Mathcmatica } & (2) & \text { Software Carousel } & (1) \\ \text { MDC II } & (1) & \text { Super Laser Spool } & (1) \\ \text { MGM Station } & (1) & \text { Trumpet } & (1) \\ \text { Mince } & (1) & \text { VTEK } & (1) \\ \text { Mol. Prescnt } & (2) & \text { Windows 286 } & (1) \\ \text { Mousc Pcrfect } & (1) & \text { Windows 386 } & (3) \\ \text { LabVicw } & (4) & \text { Windows 3.0 } & (2)\end{array}$

\section{Network Connections}

$\begin{array}{lc}\text { Existing } & \text { Estimated Total } \\ \text { FY1991 } & \text { FY1992 }\end{array}$

Lab-wide Ethernet

$$
\begin{aligned}
& \text { TCP/IP } \\
& \text { DECnet } \\
& \text { Both }
\end{aligned}
$$

Lab-wide AppleTalk

Local AppleTalk

Local 3Com

MacLink

59
34
11
50
50
0
0

63

34

11

55

50

0 
Planned activities and/or applications to be implemented during the next fiscal year in the areas of (1) scientific work stations, (2) personal computing. (3) local area networking, and (4) office automation:

1. Scientific Work Stations: We are increasing our computing capacity by adding RISC workstations to our network.

2. Personal Computing: None planned.

3. Local Area Networking: None planned.

4. Office Automation: None planned. 


\section{CHIEF OPERATIONS OFFICER (COST CENTER 202)}

\begin{tabular}{|llcl|}
\hline & & Primary Contacts & \\
Contact Person & Subject & Phone & E-Mail Address \\
Dennis Karras & All & $2-3030$ & CDC AT ANLADM1 \\
\hline
\end{tabular}

$\begin{array}{ccccc} & \text { Personal Computers and Workstations } & \\ & & & \\ & \text { Existing } & \text { Existing } & \text { Estimated Total } \\ \text { Manufacturer } & \text { FY1990 } & \text { FY1991 } & 1992 \\ \text { IBM } & 10 & 14 & 6 \\ \text { MacIntosh } & 6 & 6 & 14 \\ \end{array}$

\begin{tabular}{|cccc|}
\hline & Word Processors & & \\
& & & Estimated Total \\
Manufacturer & Existing & Existing & FY 1992 \\
$\mathrm{NBI}$ & FYl990 & FY1991 & 0 \\
\hline
\end{tabular}

\section{Software}

Word Processing/Editing

NBI (3)

Microsoft Word $5.0(6)$

WordPcrfoct

Network NBI Oasys System

Other

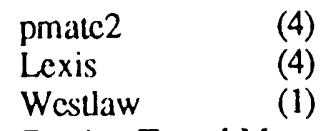

Foreign Travel Management (2)
Utilities

Microsoft Word Power Point
for Windows 3.0

Database

DBase IIIt

DBase IV

(3)

(2) 


\section{Network Connections}

Existing
FY1991

NBI Oasys

Novell
35

0
Estimated Total FY1992

35

Planned activities andlor applications to be implemented during the next fiscal year in the areas of (1) scientific work stations, (2) personal computing, (3) local area networking, and (4) office automation:

1. Scientific Work Stations: None planned.

2. Personal Computing: None planned.

3. Local Area Networking: Plan to hook up to ESHIQA Oversight Novell network, two or three terminals.

4. Office Automation: None planned. 


\section{COMPUTING AND TELECOMMUNICATIONS DIVISION (COST CENTERS 146, 245, 247, AND 296)}

\begin{tabular}{|llll|}
\hline & \multicolumn{3}{c|}{ Primary Contacts } \\
Contact Person & Subject & Phone & E-Mail Address \\
& & & \\
Jim Regula & Workstations (IBM) & $2-7622$ & B37816 AT ANLVM \\
& 3Com Networking & & \\
Dave Lifka & Workstations (Macintosh) & $2-3251$ & B36857 AT ANLCV1 \\
Sally Ott & Inventory & $2-8537$ & B11807 AT ANLVM \\
Linda Winkler & Workstations (Sun) & $2-7236$ & WINKLER@SUNGATE.CTD.ANL.GOV \\
& & & \\
\hline
\end{tabular}

\begin{tabular}{|lccc|}
\hline & Personal & Computers & and Workstations \\
& Existing & Existing & Estimated Total \\
& FY1990 & FY199] & FY1992 \\
Manufacturer & 56 & 98 & 110 \\
IBM (PC, AT, XT, PS/2) & 33 & 45 & 52 \\
Applc (II, Macintosh) & 1 & 1 & 1 \\
Compaq & 9 & 24 & 34 \\
Sun & 0 & 1 & 1 \\
Xcrox & 0 & 1 & 2 \\
Texas Instruments & 0 & 4 & 6 \\
Toshiba Laptop & 0 & 1 & 4 \\
Ncxt & 0 & 3 & 4 \\
Iris & & & \\
\hline
\end{tabular}

\section{Software}

Word Processing/Editing

MacWritc

Word

KEDIT

Text Editor

Database

DBase III Plus

Spreadsheet

Multiplan

Integrated Packages

Excel

Helix
Graphics

$\begin{array}{ll}\text { MacDraw II } & (6) \\ \text { MacPaint } & (6) \\ \text { Gem Draw } & (5) \\ \text { Superlmage } & (8)\end{array}$

Desktop Publishing

PageMaker

(3)

Project Management

MacProject II

(10)

Communication/Terminal Emulation

$\begin{array}{ll}\text { VTerm } & (4) \\ \text { Kermit } & (9) \\ \text { AZPC 2 } & (3) \\ \text { IBM PC 3270 } & (4)\end{array}$




\section{Network Connections}

$\begin{array}{lc}\text { Existing } & \text { Estimated Total } \\ \text { FY1991 } & \text { FY1992 }\end{array}$

Lab-wide Ethernet

TCP/IP

125

150

DECnet

610

675

Lab-wide AppleTalk

450

600

Local AppleTalk

Local 3Com

Planned activities and/or applications to be implemented during the next fiscal year in the areas of (1) scientific work stations. (2) personal computing, (3) local area networking, and (4) office automation:

1. Scientific Work Stations:Developing X-Windows based utilities for distributive workstations; providing access to high quality output devices.

2. Personal Computing: Implementing personal computer access to high quality color output devices (e.g. slide, transparencies).

3. Local Area Networking: Upgrade two 3Com servers to 3Com Series 500 (386) servers. Install 3+ Open operating system and eventually migrate to MS-Lanmanager. We will also be installing a network E-Mail system with a gateway to SMTPIX.400 mail protocols.

4. Office Automation: Develop experience with document conversions utilities such as Software Bridge. 


\section{DIVISION OF EDUCATIONAL PROGRAMS (COST CENTER 139)}

\begin{tabular}{|llcl|}
\hline & \multicolumn{3}{c|}{ Primary Contacts } \\
Contact Person & Subject & Phone & E-Mail Address \\
Catherine DuFour & $\begin{array}{l}\text { Workstations } \\
\text { Networks }\end{array}$ & $2-4817$ & B39548 AT ANLVM \\
\hline
\end{tabular}

\section{Personal Computers and Workstations}

Manufacturer
IBM (PC, AT, XT, PS/2)
Compaq
Club American
Tatung
Epson

Existing
FY1990
11
1
2
9
$15-20$

Existing

FY1991

Estimated Total FY1992

$\begin{array}{cr}1 & 11 \\ 1 & 1 \\ 2 & 2 \\ 9 & 9 \\ 20 & 15-20\end{array}$

15

1

2

9
$20-25$

\section{Software}

Word Processing/Editing

Word

Spreadsheet

Supercalc

Quaturo Pro

Communiction/Terminal Emulation Kcrmit

Graphics

Super Image

Harvard Graphics (2)
Desktop Publishing

Ventura

(1)

Languages/Compilers

Turbo BASIC (1)

Turbo Pascal

(1)

Database

DBase IIIt

\section{Network Connections}

Existing

FY1991

Local 3Com

\section{Estimated Total} FY1992

35 
Planned activities andlor applications to be implemented during the next fiscal year in the areas of $(1)$ scientific work stations, (2) personal computing. (3) local area networking, and (4) office automation:

1. Scientific Work Stations: None planned.

2. Personal Computing: None planned.

3. Local Area Networking: Plan to hook up to the Lab-wide network through the use of a CISCO router. We also plan to install a new E-mail system (such as CC:mail) with a gateway to recognize SMTP.

4. Office Automation: None planned. 


\section{ELECTRONICS (COST CENTER 143)}

\begin{tabular}{|llcc|}
\hline & \multicolumn{3}{c|}{ Primary Contacts } \\
Contact Person & Subject & Phone & E-Mail Address \\
& & $2-6964$ & N/A \\
Dale Travis & All & $2-6934$ & N/A \\
Lorctta Phillips & All & & \\
& & & \\
\hline
\end{tabular}

Personal Computers and Workstations

$\begin{array}{cccc} & \text { Existing } & \text { Existing } & \text { Estimated Total } \\ \text { Manufacturer } & \text { FY1990 } & \text { FY1991 } & \text { FY1992 }\end{array}$

Next 0

$\mathrm{MCI}$

EL Clone

Apple

Gatcway

IBM

Panasonic

SUN

DEC

$\begin{array}{ll}0 & 2 \\ 0 & 1 \\ 0 & 2 \\ 0 & 3 \\ 0 & 17 \\ 0 & \\ 0 & \\ 0 & \\ 0 & 7\end{array}$

\section{Software}

Word Processing/Editing Correct Grammar

PC Write

Rightword

MS Word

WordPerfoct

Wordstar

Database

DBase III

DBase IV

Paradox

(2)

(9)
(1)

(2)

Communication/Terminal Emulation

$\begin{array}{ll}\text { Brooklyn Bridge } & (4) \\ \text { Carbon Copy } & (1) \\ \text { Procom Plus } & (4) \\ \text { Reflections } & (2)\end{array}$

$\begin{array}{cc}2 & 2 \\ 1 & 1 \\ 21 & 25 \\ 3 & 5 \\ 17 & 20 \\ 1 & 1 \\ 1 & 1 \\ 4 & 6 \\ 7 & 7\end{array}$

Spreadsheet

Lotus 1-2-3

MS Excel

Quattro Pro

(1)

(1)

(9) 


\section{Software}

Graphics

DrawPerfoct

GemArline

Harvard Graphics

McDraw II

Proshow

Utility

53 various packages
(4)

(1)

(1)

(1)

(2)

Operating System

$3+$ open

Desq/View

(1)

Direct Access

(10)

DR DOS

(1)

$\mathrm{Gem} / 3$

(2)

IBM DOS

(1)

LAN Manager

MS DOS

(7)

Netware

Norton Desktop

MS Windows

(2)

Uniforth Profs

\section{Network Connections}

$\begin{array}{lc}\text { Existing } & \text { Estimated Total } \\ \text { FY1991 } & \text { FY1992 }\end{array}$

Lab-wide Ethernet

7

TCP/IP

10

Telnet

DECNet Vax

2

Mac Applctalk

5

IBM PC Clone Pathworks

Terininal Server with LAT

Cisco router

10

5

1

9
12
2
5
16
5
1

Planned activities andlor applications to be implemented during the next fiscal year in the areas of (1) scientific work stations, (2) personal computing, (3) local area networking, and (4) office automation:

1. Scientific Work Stations: None planned.

2. Personal Computing: None planned.

3. Local Area Networking: None planned.

4. Office Automation: None planned. 


\section{ENERGY, ENVIRONMENTAL, AND BIOLOGICAL RESEARCH PROGRAM ADMINISTRATION (COST CENTER 274)}

\section{Primary Contacts}

Contact Person

Austine Pctersen
Subject

MacIntosh
Phone

$2-3721$
E-Mail Address

N/A

\section{Personal Computers and Workstations}

Manufacturer

MacIntosh II

MacIntosh IIcs

Macintosh IIci

MacIntosh $1 \mathrm{Mb}$

MacIntosh Portable

Gatcway $200386 / 33 c$

MacIntosh SE 30

Howteck Scanner

Image Writer II

Lascr Writer II

Hewlett Packard Desk Jet

Hewleu Packard Scan Jel

Hewlet Packard Desk Writer

\section{Existing \\ FY1990}

\section{1}

3

2

1

0

0

1

1

2

2

0

1

1

\section{Existing \\ FY1991}

1
3
2
1
1
1
1
1
2
2
1
1
1

\section{Estimated Total FY1992}

\section{Word Processors}

$\begin{array}{lccc}\text { Manufacturer } & \text { Existing } & \text { Existing } & \text { Estimated Total } \\ \text { FY1990 } & \text { FY1991 } & 1992 \\ \mathrm{NBI} & 0 & 0 & 0\end{array}$

\section{Software}

Word Processing/Editing

Word

Graphics

Spreadsheet

Excel

(4)

Microsoft Chart (1) MacDrawII Springboard Publisher (1) MacProject II

PixelPaint (1) Canvas 2.0

Printshop

(1) Bisomess 1
(2)

(1)

(1)

(1) 


\section{Software (continued)}

Graphics

Microsoft Power Point

Cricket Graph

Manufacturer

$\begin{array}{ll}\text { Existing } & \text { Existing } \\ \text { FY1990 } & \text { FY1991 }\end{array}$

Estimated Total FY1992

Howteck Scanner

Image Writer II

Laser Writer II

Hewlet Packard Desk Jet

Hewlet Packard Scan Jet

Hewleu Packard Desk Writer

1
2
2
0
1
1

1

2

2

1

1

1

Planned activities and/or applications to be implemented during the next fiscal year in the areas of $(1)$ scientific work stations, (2) personal computing, (3) local area networking, and (4) office automation:

1. Scientific Work Stations: None planned.

2. Personal Computing: Software upgrade and improved peripheral equipment.

3. Local Area Networking: None planned.

4. Office Automation: None planned. 


\section{ENERGY, ENVIRONMENTAL, AND BIOLOGICAL RESEARCH PROGRAM DIRECTION (COST CENTER 174)}

\section{Primary Contacts}

Contact Person

Subject

Phone

$\begin{array}{lll}\text { All } & 2-4469 & \text { harkins@anlcmt.anl.gov } \\ \text { All } & 2-6230 & \text { B27323 At ANLEES }\end{array}$

Marian Harkins

Denise Voss

\section{E-Mail Address}

B27323 At ANLEES

\section{Personal Computers and Workstations}

Manufacturer

IBM (PC, AT, XT, PS/2)

Apple (II, Macintosh)

Compaq

AST (286, 386 SX clones)
Existing
FY1990

3

2

1
Existing

FY1991

4
2
1
3

Estimated Total FY1992

\section{Word Processors}

Manufacturer

NBI

\section{Existing \\ FY1990}

1
Existing
FY1991

0

\section{Estimated Total FY 1992}
4
2
1

Software

Word Processing/Editing

WordPerfect

Microsoft Word 4.0

Correct Grammar

TeachText

(1)

(2)

N/A

(2)
Graphics

Deltagraph 1.5c N/A MacDraft N/A

SuperPaint 1.1MS N/A

Communication/Terminal Emulation

Reflection 4+ (1)

NCSA Telnet 2.3 (2)

Red Ryder 10.0 N/A

Red Ryder 10.3 N/A

Tops DA

(2)

Utilities

Project Management

Microsoft Excel (2)

Norton Utilities (2) 


\section{Software (continued)*}

Other

Apple File Exhange

DCA Conversion 1.5

Disk First Aid

(2)

Edit

Font Harmony

Hard Disk Formatter 3.27.3

Install Keypad

Layout Plus

MacWelcome 1.4

(2)

Offline 2.0

PrintMonitor

RamDisk

Speed Disk

Total Recall 1.2.3 (2)

Virus Rx
Auto UnStuffIt Installer 1.5

Dialog Editor

DiskFit 2.0

Expressionist

Font/DA Mover (2)

Hard Disk Formatter 3.27.4

Installer

MacLink Plus/PC

Microsoft Excel Setup

PackIt

Profiler

Sound Mover 1.3

Start Tops

$\operatorname{tn} 3270$
BINHEX 4.0

Disinfectant 1.8

D S Backup 4.2

Font \& Sound Valet

FonU/DA Mover 3.8

HD Backup 1.6

Interferon 3.1

MacTREE Plus

Oasis 2.0

PrintDEX 1.4e

QM Forms

Sound $>$ snd $1.2 e$

Stuffit 1.5.1

UnStufftt 1.5.1

* Unless otherwise noted, copies of individual packages was unavailable.

Planned activities andlor applications to be implemented during the next fiscal year in the areas of (1) scientific work stations, (2) personal computing. (3) local area networking, and (4) office automation:

1. Scientific Work Stations: None planned.

2. Personal Computing: May upgrade/replace 286 machine. May acquire portable Macintosh or clone.

3. Local Area Networking: None planned.

4. Office Automation: Labelwriter. 


\section{ENGINEERING PHYSICS (COST CENTERS 115, 117, AND 211)}

\section{Primary Contacts}

$\begin{array}{llll}\text { Contact Person } & \text { Subjeci } & \text { Phone } & \text { E-Mail Address } \\ \text { Roland Armani } & \text { All } & 2-4855 & \text { N/A }\end{array}$

\section{Personal Computers and Workstations}

Manufacturer

IBM (PC, AT, XT, PS/2)

Apple (II, Macintosh)

Compaq

Sun

Club American

AST Rescarch

MicroStar

Radio Shack

Others

Gatcway

Existing
FY1990
42
15
7
4
2
2
9
4
5
0

\section{Existing \\ FY1991}

$42 \quad 53$

$15 \quad 34$

$\begin{array}{ll}7 & 17\end{array}$

4 11

21

22

925

42

21

\begin{tabular}{ll} 
& 31 \\
\hline
\end{tabular}
Estimated Total FY1992

55

36

18

13

1

2

5

2

23

\section{Word Processors}

Manufacturer

Existing

FY1990

Existing

FY1991

Estimated Total

FY 1992

Exxon/Vydoc

NBI

2
2

0

0

Software

Word Processing/Editing

MacWrite

Word

WordPcrfect

WordStar

PC Write

Database

$$
\text { DBasc III Plus }
$$

DBase IV

Integrated Packages

Exccl
Graphics

MacDraw II
MacPaint
Freelance
SuperImage
Harvard Graphics
Graphwriter

Spreadsheet

Multiplan

Lotus 1-2-3

Communication/Terminal Emulation Kermit XTalk 


\section{Software (continued)}

Languages/Compilers

Fortran

C

Turbo Pascal

Basic

Assembly

$C A E / C A D$

AutoCAD
(3)

(1)
Utility

Norton Utilities

(3)

Mace

Norton Commander

$\mathrm{X}$-Tree

\section{Network Connections}

$\begin{array}{lc}\text { Existing } & \text { Estimated Total } \\ \text { FY1991 } & \text { FY1992 }\end{array}$

Lab-wide Ethernet

TCP/IP

44

53

Local 3Com

6

6

Planned activities andlor applications to be implemented during the next fiscal year in the areas of (1) scientific work stations, (2) personal computing, (3) local area networking, and (4) office automation:

1. Scientific Work Stations: Add CAD stations as needed and continue upgrade of older equipment.

2. Personal Computing: Maintain a high level of computing competency in engineering personal computing through improvements in software and computer capabilities.

3. Local Area Networking: Upgrade the Division servers to accommodate more users and implement the use of networking between scientific managers and Division administration.

4. Office Automation: None planned. 


\section{ENGINEERING RESEARCH (COST CENTERS 171 AND 271)}

\section{Primary Contacts}

$\begin{array}{llll}\text { Contact Person } & \text { Subject } & \text { Phone } & \text { E-Mail Address } \\ \text { Ronald Scharping } & \text { All } & 2-4490 & \text { B11898 @ANLVM }\end{array}$

Personal Computers and Workstations

Manufacturer

IBM (PC, AT, XT, PS/2)

$\begin{array}{ll}\text { Existing } & \text { Existing } \\ \text { FY1990 } & \text { FY1991 }\end{array}$

6

11

\section{Estimated Total FY1992}

11

\section{Word Processors}

Manufacturer

NBI ICU

NBI Workstations

\section{Existing \\ FY1990}

1

6
Existing

FY1991

1

6

\section{Estimated Total} FY 1992

0

0

\section{Software}

Word Processing/Editing

WordStar

Graphics

WordPerfect

(6)

Freelance

(1)

Database

DBasc III Plus (1)

DBase IV

Desktop Publishing

Ventura

(1)

Spreadsheet
Lotus 1-2-3
Quattro Pro
(1)
(1)

\section{Network Connections}

\section{Existing \\ FY1991}

Local 3Com

\section{Estimated Total FY1992}

2 
Planned activities and/or applications to be implemented during the next fiscal year in the areas of (1) scientific work stations, (2) personal computing. (3) local area networking, and (4) office automation:

1. Scientific Work Stations: None planned.

2. Personal Computing: None planned.

3. Local Area Networking: Establish LAN within and between administrative offices. Connect LAN to existing building Ethernet cable and node.

4. Office Automation: The network in item 3 will support the goal of connectivity between office personnel. 


\section{ENVIRONMENT, SAFETY, AND HEALTH/QUALITY ASSURANCE OVERSIGHT (COST CENTER 333)}

\section{Primary Contacts}

Contact Person

Subject

Phone

E-Mail Address

Dan Miller

All

$2-5775$

N/A

\section{Personal Computers and Workstations}

$\begin{array}{lcc}\text { Existing } & \text { Existing } & \text { Estimated Total } \\ \text { FY1990 } & \text { FY1991 } & \text { FY1992 }\end{array}$

Manufacturer

Existing

FY1991

FYI992

IBM (PC, AT, XT, PS/2)

Gatcway 486-33

Gatcway 485-25

Gatcway 386-33

Gatcway 386-25

Apple IIci

1
0
0
0
0
0

5
5
3
3
7
1

5
5
3
3
7
1

\section{Word Processors}

Manufacturer

NBI

\section{Existing}

FY1990

2
Existing

FY1991

0

\section{Estimated Total} FY 1992 


\section{Software}

Word Processing/Editing

WordPerfect

(18)

Communication/Terminal Emulation

Lan/PC $\quad 15$ users

Lan/MAC 15 users

Procomm Plus (3)

Spreadsheet

Lotus 1-2-3

Excel

(4)

(5)

\section{Database}

dBase IV 9 users

Foxpro 2.0 Lan 250 usera

SQLbase 5 users

Network SQL 250 users

Emerald Bay 2 users

Graphics

$\begin{array}{ll}\text { Powerpoint } & \text { (4) } \\ \text { Harvard } & \text { (2) } \\ \text { Corel Draw } & \text { (1) }\end{array}$

Utility

SQLWindows 5 users

dAnalyst

(1)

Codebase ++ (1)

Phar Lap 386 (1)

Borland C++ (1)

Netware C 386 (1)

Perform Pro (2)

Vulcan

(1)

Fox Distribution (1)

\section{Network Connections}

Existing

FY1991

Novell

DaVinci

Nctware Access
250

100

15
Estimated Total FY1992

250

100

15 
Planned activities and/or applications to be implemented during the next fiscal year in the areas of (1) scientific work stations, (2) personal computing. (3) local area networking, and (4) office automation:

1. Scientific Work Stations: None planned.

2. Personal Computing: None planned.

3. Local Area Networking: None planned.

4. Office Automation: None planned. 


\section{ENVIRONMENT, SAFETY, AND HEALTH DIVISION-DIVISION OFFICE} (COST CENTER 235)

\begin{tabular}{|lccc|} 
& & Primary & Contacts \\
Contact Person & Subject & Phone & E-Mail Address \\
Dolores Ray & All & $2-5640$ & N/A \\
\hline
\end{tabular}

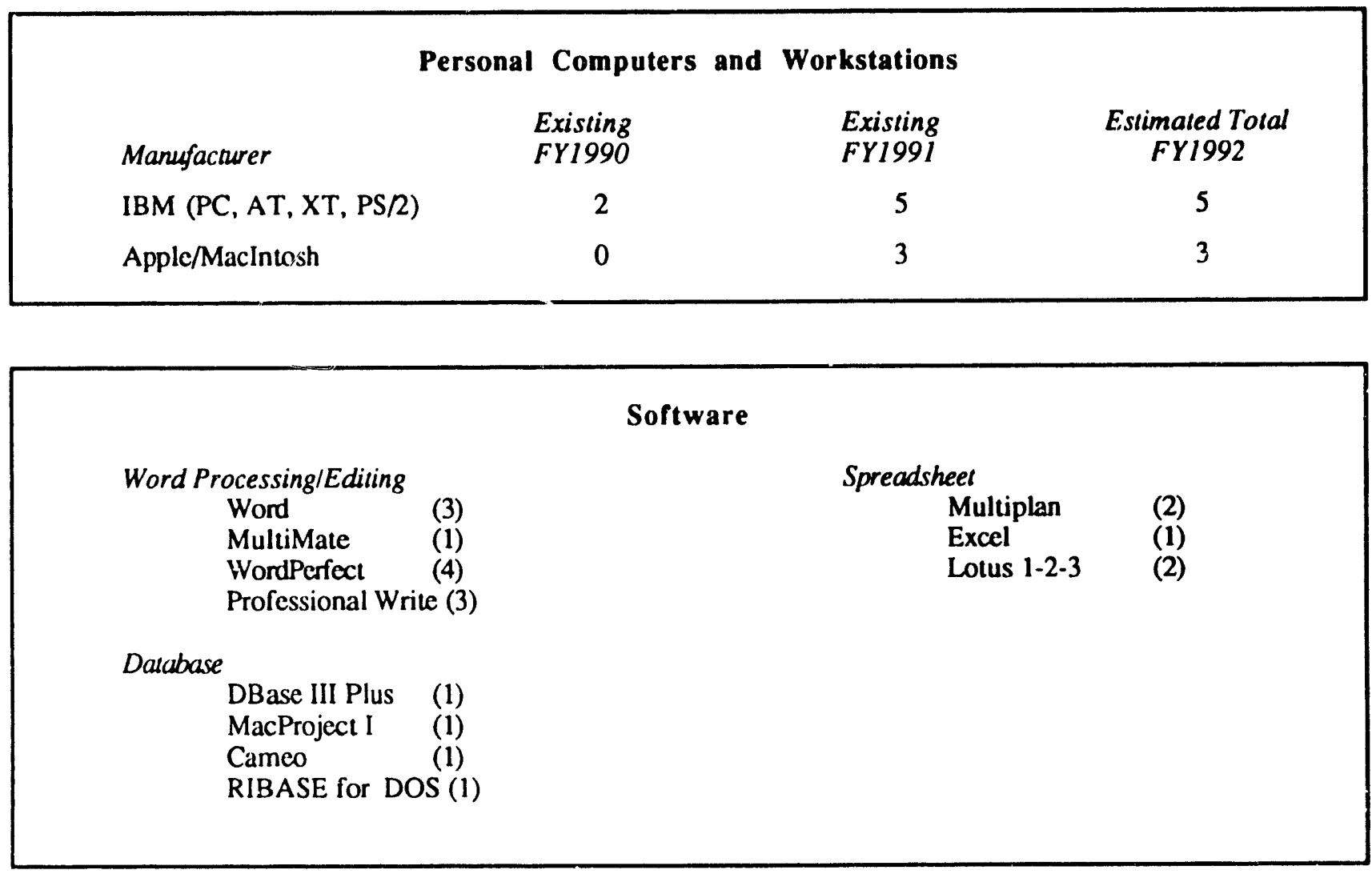


Planned activities and/or applications to be implemented during the next fiscal year in the areas of $(1)$ scientific work stations, (2) personal computing, (3) local area networking, and (4) office automation:

1. Scientific Work Stations: None planned.

2. Personal Computing: None planned.

3. Local Area Networking: All PCS will be added to the ESH 3COM Local area network.

4. Office Automation: Nore planned. 


\section{ENVIRONMENT, SAFETY, AND HEALTH DIVISION- DOSIMETRY AND ANALYTICAL SERVICES (EXTERNAL DOSIMETRY) (COST CENTER 235)}

\begin{tabular}{|llll|}
\hline & & Primary Contacts & \\
Contact Person & Subject & Phone & E-Mail Address \\
Dolores Ray & All & $2-5640$ & N/A \\
\hline
\end{tabular}

\begin{tabular}{lcccc} 
& Personal Computers & and Workstations & \\
& Existing & Existing & Estimated Total \\
Manufacturer & FY1990 & FY1991 & FY1992 \\
IBM (PC, AT, XT, PS/2) & 2 & 6 & 6 \\
\hline
\end{tabular}

\section{Software}

Word Processing/Editing

Professional Write (7)

Database

DBase III Plus (7)

Planned activities and/or applications to be implemented during the next fiscal year in the areas of (1) scientific work stations, (2) personal computing. (3) local area networking, and (4) office automation:

1. Scientific Work Stations: None planned.

2. Personal Computing: None planned.

3. Local Area Networking: All workstations are to be added to the ESH 3COM local area network.

4. Office Automation: None planned. 


\section{ENVIRONMENT, SAFETY, AND HEALTH DIVISION- DOSIMETRY AND ANALYTICAL SERVICES (INTERNAL DOSIMETRY) (COST CENTER 235)}

\section{Primary Contacts}

$\begin{array}{llll}\text { Contact Person } & \text { Subject } & \text { Phone } & \text { E-Mail Address } \\ \text { Dolores Ray } & \text { All } & 2-5640 & \text { N/A }\end{array}$

Personal Computers and Workstations

Manufacturer

IBM (PC, AT, XT, PS/2)

$\begin{array}{cc}\text { Existing } & \text { Existing } \\ \text { FY1990 } & \text { FY1991 } \\ 4 & 10\end{array}$

\section{Software}

Word Processing/Editing KEDIT

WordPcrfect

Lotus 1-2-3

Communication/Terminal Emulation

Kermit

(3)
Database

$\begin{array}{ll}\text { DBase III Plus } & (3) \\ \text { DBase IV } & (4)\end{array}$

Languages/Compilers QuickBasic

Other
Estimated Total FY1992

10

Network Connections

Existing

FY1991
Estimated Total FY1992

Lab-wide Ethernet DECnet

2

2

Local 3Com

(1) 
Planned activities andlor applications to be implemented during the next fiscal year in the areas of $(1)$ scientific work stations, (2) personal computing. (3) local area networking, and (4) office automation:

1. Scientific Work Stations: None planned.

2. Personal Computing: None planned.

3. Local Area Networking: All workstations are to be added to the ESH 3COM local area network.

4. Office Automation: None planned. 


\section{ENVIRONMENT, SAFETY, AND HEALTH DIVISION-ENVIRONMENTAL PROTECTION (COST CENTER 235)}

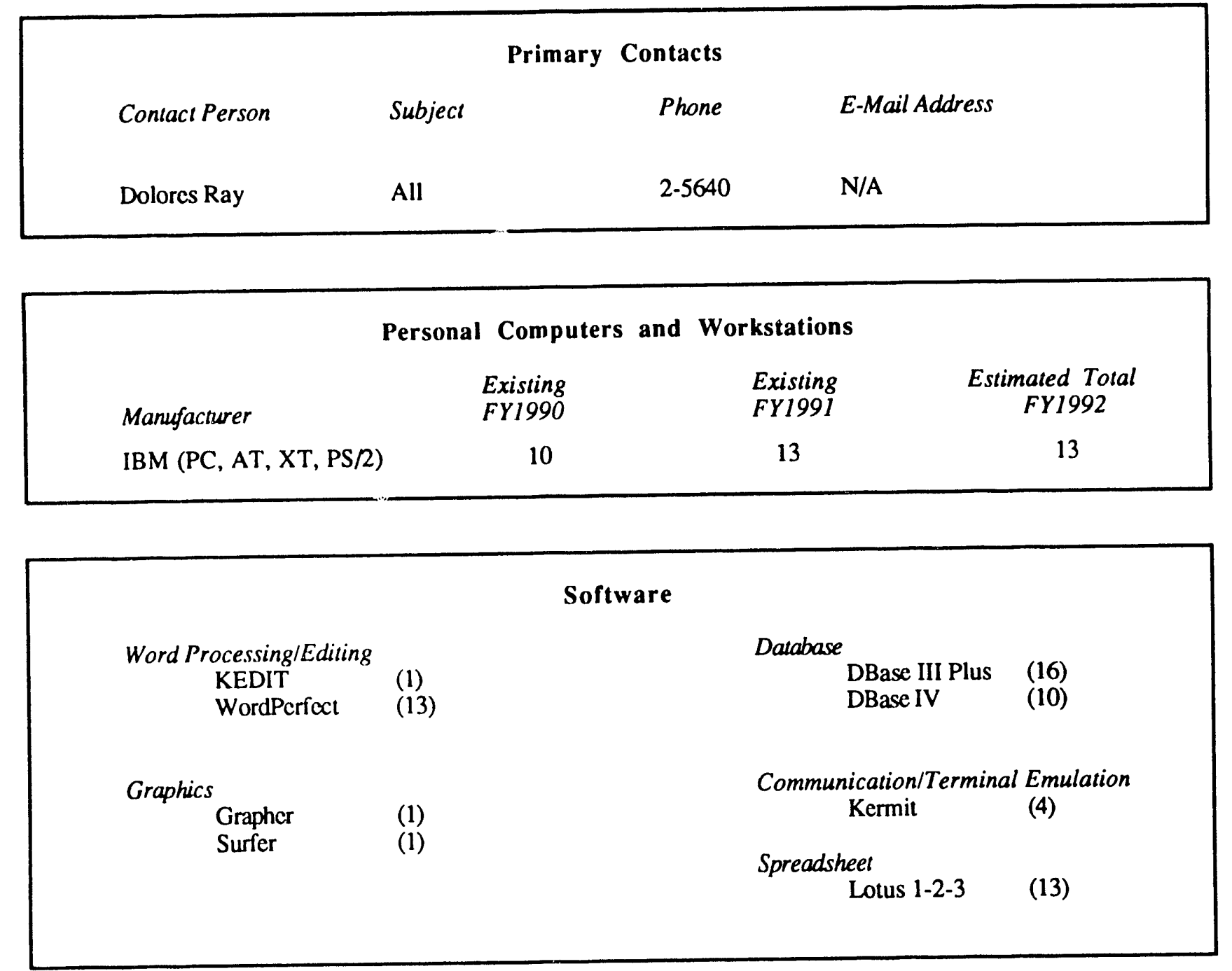

\begin{tabular}{|c|c|c|}
\hline \multicolumn{3}{|c|}{ Network Connections } \\
\hline & $\begin{array}{l}\text { Existing } \\
\text { FY1991 }\end{array}$ & $\begin{array}{c}\text { Estimated Total } \\
\text { FY1992 }\end{array}$ \\
\hline Local 3Com & 13 & 13 \\
\hline
\end{tabular}


Planned activities andlor applications tc be implemented during the rext fiscal year in the areas of (1) scientific work stations, (2) personal computing, (3) local area networking, and (4) office automation:

1. Scientific Work Stations: None planned.

2. Personal Computing: None planned.

3. Local Area Networking: None planned.

4. Office Automation: None planned. 


\section{ENVIRONMENT, SAFETY, AND HEALTH DIVISION-HEALTH PHYSICS (COST CENTER 235)}

\begin{tabular}{|lrrr|}
\hline & & Primary Contacts & \\
Contact Person & Subject & Phone & E-Mail Address \\
Dolores Ray & All & $2-5640$ & N/A \\
\hline
\end{tabular}

Personal Computers and Workstations

$\begin{array}{lccc} & \text { Existing } & \text { Existing } & \text { Estimated Total } \\ \text { Manufacturer } & \text { FY1990 } & \text { FY1991 } & \text { FY1992 } \\ \text { IBM (PC, AT, XT, PS/2) } & 0 & 7 & 7 \\ \text { Portablcs/Lap Tops } & 0 & 6 & 6\end{array}$

\section{Software}

Word Processing/Editing

PFS Write

WordPerfect

(6)

(2)
Database

DBase III Plus

(4)

Communication/Terminal Emulation

Kermit

(1)

Planned activities andlor applications to be implemented during the next fiscal year in the areas of (1) scientific work stations, (2) personal computing, (3) local area networking, and (4) office automation:

1. Scientific Work Stations: None planned.

2. Personal Computing: None planned.

3. Local Area Networking: None planned.

4. Office Automation: None planned. 


\section{ENVIRONMENT, SAFETY, AND HEALTH DIVISION-INDUSTRIAL HYGIENE (COST CENTER 235)}

\section{Primary Contacts}

$\begin{array}{llll}\text { Contact Person } & \text { Subject } & \text { Phone } & \text { E-Mail Address } \\ \text { Dolores Ray } & \text { All } & 2-5640 & \text { N/A }\end{array}$

\section{Personal Computers and Workstations}

Manufacturer

IBM (PC, AT, XT, PS/2)

Hewleu Packard

$\begin{array}{cc}\text { Existing } & \text { Existing } \\ \text { FY1990 } & \text { FY1991 } \\ 4 & 4 \\ 1 & 1\end{array}$

Estimated Tolal FY1992

4

1

\section{Software}

Word Processing/Editing WordPerfect

Zenword

(1)

Spreadsheet

$$
\text { Lotus 1-2-3 }
$$

Communication/Terminal Emulation

Kermit

XTALK

SIMPC

(2)

(2)

(2)

CAE/CAD

CADD II

FormTool

(1)

(2)
Databases

DBase III Plus (5)

Cameo

Gloves

CCInfo

Clothing

(1)

(1)

(1)

(1)

Languages/Compilers

Basic

Utility

PC Tools

Enercharts

(5)

(1)

FastBack

(1)

Metsoft

(3)

E

Software Bridge

Other

Portacount

(1) 


\section{Network Connections}

Existing Estimated Total

FY1991

FY1992

Local 3Com

5

5

Planned activities andlor applications to be implemented during the next fiscal year in the areas of (1) scientific work stations, (2) personal computing. (3) local area networking, and (4) office automation:

1. Scientific Work Stations: None planned.

2. Personal Computing: None planned.

3. Local Area Networking: None planned.

4. Office Autonation: None planned. 


\section{ENVIRONMENT, SAFETY, AND HEALTH DIVISION-SAFETY ENGINEERING SECTION (COST CENTER 234)}

\begin{tabular}{|lcrc|}
\hline & & Primary & Contacts \\
Contact Person & Subject & Phone & E-Mail Address \\
& All & $2-5640$ & N/A \\
Dolores Ray & & & \\
\hline
\end{tabular}

Personal Computers and Workstations

Manufacturer

$\begin{array}{ll}\text { Existing } & \text { Existing } \\ \text { FY1990 } & \text { FY1991 }\end{array}$

Estimated Total FY1992

IBM (PC, AT, XT, PS/2)

Gatcway

32

2

$\begin{array}{lll}3 & 2 & 2 \\ 0 & 1 & 1\end{array}$

Software

Word Processing/Editing

WordPerfect

(3)

Database

DBase III Plus (1)

Graphics

Harvard Graphics (1)

Utility

PC Tools

(1)

Spreadsheet

Communication/Terminal Emulation

Excel

(1)

(1) 
Planned activities andlor applications to be implemented during the next fiscal year in the areas of $(1)$ scientific work stations. (2) personal computing. (3) local area networking, and (4) office automation:

1. Scientific Work Stations: None planned.

2. Personal Computing: None planned.

3. Local Area Networking: All workstations are to be added to ESH 3COM local area network.

4. Office Automation: None planned. 


\section{ENVIRONMENT, SAFETY, AND HEALTH DIVISION-TRAINING AND QUALITY ASSURANCE (COST CENTER 235)}

Primary Contacts

Contact Person Subject Phone E-Mail Address

Dolores Ray

All

$2-5640$

N/A

\section{Software}

Word Processing/Editing

WordPerfect

(3)

Professional Write (1)
Graphics

Harvard Graphics (1)

Energraphics
Estimated Total FY1992

4

Planned activities undlor applications to be implemented during the next fiscal year in the areas of (1) scientific work stations, (2) personal computing. (3) local area networking, and (4) office automation:

1. Scientific Work Stations: None planned.

2. Personal Computing: None planned.

3. Local Area Networking: All workstations are to be added to ESH 3COM local area network.

4. Office Automation: None planned. 


\section{ENVIRONMENTAL ASSESSMENT AND INFORMATION SCIENCES DIVISION (COST CENTER 165)}

\begin{tabular}{|c|c|c|c|}
\hline \multicolumn{4}{|c|}{ Primary Contacts } \\
\hline Contact Person & Subject & Phone & E-Mail Address \\
\hline $\begin{array}{l}\text { Doe Seymour } \\
\text { Lou Kvitek } \\
\text { Dick Eagan } \\
\text { James Renz }\end{array}$ & $\begin{array}{l}\text { IBM } \\
\text { Sun Workstations } \\
\text { Sun Networks } \\
\text { PC and Mac } \\
\text { Networks }\end{array}$ & $\begin{array}{l}2-8023 \\
2-3132 \\
2-3435 \\
2-4650\end{array}$ & $\begin{array}{l}\text { seymour@athens.eid.anl.gov } \\
\text { kvitek@athens.ees.anl.gov } \\
\text { eagan@athens.ces.anl.gov } \\
\text { renz@ athens.eid.anl.gov }\end{array}$ \\
\hline
\end{tabular}

\section{Personal Computers and Workstations}

Manufacturer

IBM (PC, AT, XT, PS/2)

Apple (II, Macintosh)

Compay

Sun

Zenith

AST Rescarch

Texas Instruments

Toshiba

Epson

PC Designs

Compu Add

$\mathrm{NeXT}$

NEC

Visual Tech

Gatcway 2000

Existin
FY199
215
93
31
47
28
7
1
12
2
5
3
3
6
18
0

Estimated Total FY1992

Existin
215
100
50
57
28
7
0
15
2
5
3
5
10
18
50

220

110

60

70

28

7

0

20

2

5

3

7

15

18

75 


\section{Software}

Word Processing/Editing

MacWrite

Word

WEDIT

Word Perfect

Word Star

Norton

Database

DBase

Q \& A

Paradox

Project Management

MacProject Il

Timeline

Languages/Compilers

MS Fortran

Lahey Fortran

Turbo Pascal

Turbo C

MS C

Utility

Norton

MS Windows

Graphics

MacDraw II

Gem Draw

Harvard Graphics

Kaleidagraph

Graphic

SuperImage

Cricket Graph

Desktop Publishing

PageMaker

Legend

Integrated Packages

Excel

Lotus 1-2-3

Communication/Terminal Emulation

VTerm

(7)

Procomm

(50)

VersaTerm

Red Ryder

Relay Gold

Kermit

SmarTerm

(9)

(50)

CAE/CAD

AutoCAD

MacDraft

(1)

\section{Network Connections}

\section{Existing \\ FY1991}

Lab-wide Ethernet

TCP/IP

DECnct

Both

Lab-wide AppleTalk

Local 3Com

Novell

300
3
3
120
150
0

\section{Estimated Total} FY1992

375

3

3

0

200 
Planned activities and/or applications to be implemented during the next fiscal year in the areas of (1) scientific work stations, (2) personal computing. (3) local area networking, and (4) office automation:

1. Scientific Work Stations: Developing $X$-Windows based utilities and visualization applications.

2. Personal Computing: Providing Division-wide electronic mail. Integrating all PCs into local area nelworks, including remote offices.

3. Local Area Networking: Converting all local area networks to Novell.

4. Office Automation: None planned. 


\section{ENVIRONMENTAL RESEARCH DIVISION (COST CENTER 149)}

\begin{tabular}{llcl|} 
& & Primary & Contacts \\
Contact Person & Subject & Phone & E-Mail Address \\
Terry Pawlisz & All & $2-4487$ & terry_pawlisz@mctgate.mct.anl.gov \\
& & & \\
\hline
\end{tabular}

Manufacturer
IBM (PC, AT, XT, PS/2)
Apple (II, Macintosh)
Zenith
ComputAdd
Gateway
Sun

Personal Computers and Workstations

Existin
FY1990
11
6
6
0
0
0

\section{Existing \\ FY1991}

\begin{tabular}{cc|}
10 & 12 \\
65 & 72 \\
3 & 3 \\
2 & 2 \\
1 & 1 \\
1 & 1 \\
\hline
\end{tabular}

\begin{tabular}{|cccc|}
\hline & Word Processors & (Portable/Lap Tops) & \\
& & & \\
Manufacturer & Exising & Existing & Estimated Total \\
& FY.990 & FY1991 & FY1992 \\
Zenith & & 1 & 1 \\
Dauphin (386) & & 1 & 1 \\
Toshiba & 2 & 2 \\
MacIntosh & 1 & 1 \\
Cambridge & 1 & 1 \\
\hline
\end{tabular}




\section{Software}

Word Processing/Editing

MacWrite

Microsoft Word

WordPerfoct

Writc Now

Database

DBase III+

Filcmakcr (II, Pro)

DBase IV

(2)

Genifer

$\mathrm{dBXL}$

Utility

Systat

Norton Utilitics

Symant c Utilitics

(1)

Communica'ion/Terminal Emulation

Ver. aTcm (reg, pro)

Red Ryoier

NCSA Telnet (site-wide license)

Presentation Packages

Power Point

Integrated Packages

EXSYS

EXSYS Pro

Other

MORE II

Calendar Maker

Smart Forms

Adobe Type Manager

The Big Thesaurus

Tenneloc

(3) Smart Labels

(5) TOPS

(2) DiskFit

(1) Math Type

(1) End Note +

Plink 86

(1) Level 5

(1) Word Exchange

Mac Link Plus Translators (1)
Laplink

Micro Planner
Graphics

$\begin{array}{ll}\text { MacDraw II } & (10) \\ \text { MacDraft } & (2) \\ \text { Delta Graph } & (1) \\ \text { SuperPaint } & (2) \\ \text { Kaleidagraph } & (5)\end{array}$

Spreadsheet

Lotus 1-2-3 (2)

Microsoft Excel (30)

Geographic Information Systems

GRASS

(1)

ARC-INFO

(1)

Languages/Compilers

Fortran

MacFORTRAN (1)

Quicksi ver (1)

A/UX

Basic

(1)

(1)

$C A D$

AutoCAD 386

(1)

SUM II

(8)

(7) Fast Forms

(7) Fast Forms
(3) Expressionist

(1)

(4) Image Grabber

Ortec

(2)

(1) 4th Dimension (1)

(1) Camera (1)

(1) PC Partner (1)

(0)
$(2)$
$1)$
$(2)$
$5)$

$(2)$
$30)$

stems
$1)$
$1)$

(1) 


\section{Network Connections}

$\begin{array}{lc}\text { Existing } & \text { EstimatedTotal } \\ \text { FY1991 } & \text { FY1992 }\end{array}$

Lab-wide Ethernet

TCP/IP

DECnet

3

3

7

9

Planned activirties and/or applications to be implemented during the noxt fiscal year in the areas of (1) scientific work stations, (2) personal computing. (3) local networking, and 14) office automation:

1. Scientific Work Stations: Developing Geographic Information Systems using a SUN workstation.

2. Personal Computing: Develop experience in creating compatibility with several machine types (i.e. IBM, MacIntosh, SUN).

3. Lucal A rea Networking: Install AlisaSho"e software on teh VAX to improve communication and peripheral sharing with divisional Appletalk network. Upgrade Quic'.Mail E-Mail software to version 2.5 for compatibility with MacIntosh System 7.

4. Office Automation: Develop experience with document conversion utilities such as MacLink Plus Translators. 
FACILITIES PLANNING/ENGINEERING (COST CENTER 512)

\begin{tabular}{|llrl|}
\hline & & Primary Contacts & \\
Contact Person & Subject & Phone & E-Mail Address \\
Vytcnis Milunas & All & $2-7336$ & N/A \\
\hline
\end{tabular}

\section{Personal Computers and Workstations}

\section{Manufacturer}

IBM (PC, AT, XT, PS/2)

Compaq

Epson

AT\&T

Mitac

SSD-EL

Hewlet Packard Vectras

\section{Existing}

FY1990

20
5
0
0
0
0
0

Existing
FYl991

19

19
3

3
6

6

1

5

0

\section{Estimated Total FY1992}

$1 y$
3
20
0
0
10
2

\section{Word Processors}

$\begin{array}{lccc}\text { Maxisfing } & \begin{array}{c}\text { Existing } \\ \text { FY1990 }\end{array} & \text { FY1991 } & \begin{array}{c}\text { Estimated Total } \\ \text { FY } 1992\end{array} \\ \text { NBI } & 13 & 5 & 0\end{array}$

* NBI workstations and 8 NBI connections to IBM Personal Computers

\section{Software}

Word Processing/Editing

Multimate

Word

VEDIT

WordStar

WordPerfoct

Dalabase

DBase III Plus

DBaseIV

RBase

(24)

(1)

(1)

(8)

(3)

(7)

(3)
Graphics

Harvard Graphics

Perform

Forwork

Draw Perfoct

(1)

(1)

(1)

(1)

Spreadsheet

Multiplan

Lotus 1-2-3 


\section{Software (continued)}

Project Management

Primavera

Propath

Frimictime

Micosoft Project (1)

Parade

Cambridge Analyst (1)

Advanced Pro-Path 6 (2)

\section{CAE/CAD}

AutoCAD AEC Arch

DesignCAD

AutoCAD AEC Mech

AutoSketch

MathCAD

AutoCAD 386

AutoCAD

ASG Core

ASG Architectural

ASG Mechanical

ASG Plumbing

ASG Structural

ASG 2D Piping

ASG 3D Piping

Auto Manager

Autoplot II
(1)

(1)
(6)

(1)

(G)

(1)

(1)

(7)

(1)

(6)

(6)

(6)

(6)

(1)

(1)

(1)

(7)

(6)
Languages/Compilers

Fortran

(1)

Utility

$$
\begin{array}{ll}
\text { XTREE } & (1) \\
\text { QDOS } & (4) \\
\text { Norton Utilities } & (13) \\
\text { Norton Commander (10) } \\
\text { FFM }
\end{array}
$$

Other

Emcee

386 Max Professional (10)

OSHA Fast Regs.

(1)

Micro-Doe 2

Procomm

(1)

(4)

\section{Network Connections}

\section{Existing}

FY1991

Lab-wide Ethernet

TCP/IP

Local 3Com
None
20

\section{Estimated Total FY1992}

1

60 
Planned activities and/or applications to be implemented during the next fiscal year in the areas of (1) scientific work stations, (2) personal computing, (3) local area networking, and (4) office automation:

1. Scientific Work Stations: None planned.

2. Personal Computing: Additional computers for new and existing staff. Replacement of old CGA monitors to VGA standard.

3. Local Area Networking: Growth of PC network and implementation of facilities drawing management system.

4. Office Automation: Switch from NBI word processing to PC based network. 


\section{FIRE DEPARTMENT (COST CENTER 236)}

\begin{tabular}{|llll|}
\hline & \multicolumn{3}{c|}{ Primary } \\
\hline & Subject & Phone & E-Mail Address \\
Contact Person & Workstations & $2-6136$ & N/A \\
Cynthia Hijuclos & & \\
\hline
\end{tabular}

Personal Computers and Workstations

$\begin{array}{lccc} & \text { Existing } & \text { Existing } & \text { Estimated Total } \\ \text { Manufacturer } & \text { FY1990 } & \text { FY1991 } & \text { FY1992 } \\ \text { IBM (PC, AT, XT, PS/2) } & 2 & 3 & 3 \\ \text { Apple (II, Macintosh) } & 1 & 1 & 1\end{array}$

\section{Software}

Word Processing/Editing

MultiMate

WordPerfect

(1)

Database

DBase III

DBase IV

CAMEO

FireProof (FMS)

(1)

(1)

(1)
Graphics

Microsoft Chart (1)

CAE/CAD

ACAD

(1)

Tracking System (1) 
Planned activities andlor applications to be implemented during the next fiscal year in the areas of (1) scientific work stations, (2) personal computing, (3) local area networking, and (4) office automation:

I. Scientific Work Stations: None planned.

2. Personal Computing: Initiate use of CAMEO System designed by the National Ocear: $c$ and Atmospheric Adm. and the U.S. Environmental Protection Agency to assist in enhancing our preexisting prefire planning program.

3. Local Area Networking: Networking with Emergency Management Oficer's Office on CAMEO System.

4. Office Automation: None planned. 


\section{HEALTH DEPARTMENT (COST CENTER 148)}

\section{Primary Contacts}

Contact Person

Subject

Phone

E-Mail Address

Ken Vasallo

All

$2-2804$

N/A

Personal Computers and Workstations

Manufacturer

IBM (PC, AT, XT, PS/2)
Existing

FY1990

10
Existing

FY1991

18
Estimakd Total FY1992

18

\section{Software}

Word Processing/Editing

Multimate

(3)

WordStar

(4)

Spreadsheets

SuperCalc

Lotus 1-2-3

(1)

(1)

Database

DBase IV

(2)

Informix

(1)

Clipper

(1)

Utility

SideKick
SideKick Plus
Norton 4

(2)

(1)

Norton 4

Communication/Terminal Emulation

Kermit

(2)

IBM PC3270

(7)

Other

Star Exchange (1)

Free Link Micro 8)

Stedman's Med. (1)

IBM DOS

DeFlow

(8)

(1) 
Planned activities and/or applications to be implemented during the next fiscal year in the areas of (I) scientific work stations. (2) personal computing. (3) local area networking, and (4) office automation:

1. Scientific Work Stations: None planned.

2. Personal Computing: Add new PS/2 Workstations to Network.

3. Local Area Networking: Internetwork bridge between $H R$ and $M D$.

4. Office Automation: Burcode, check in process, direct entry of physical results. 


\section{HIGH ENERGY PHYSICS (COST CENTER 137)}

\begin{tabular}{|llll|}
\hline & \multicolumn{3}{c|}{ Primary Contacts } \\
Contact Person & Subject & Phone & E-Mail Address \\
Jim Schlercth & All & $2-6281$ & JLSHEP@ANLHEP \\
\hline
\end{tabular}

\begin{tabular}{|lccc|}
\hline & Personal Computers & and & Workstations \\
& Existing & Existing & Estimated Total \\
Manufacturer & FY1990 & FY1991 & FY1992 \\
IBM (PC, AT, XT, PS/2) & 14 & N/A & N/A \\
Apple (II, Macintosh) & 20 & N/A & N/A \\
Compaq & 3 & 0 & 0 \\
VAXstation 2000 & 0 & 3 & 3 \\
VAXstation 3000 & 3 & N/A & N/A \\
Hyundai & 2 & 0 & 1 \\
DECstation & 3 & & \\
& & & \\
\hline
\end{tabular}

\begin{tabular}{|lccc|}
\hline & Word Processors & & \\
& Existing & Existing & Estimated Total \\
Manufacturer & FY1990 & FY1991 & N/A \\
Exxon/Vydoc & 1 & N/A & N/A \\
NBI & 2 & N/A & 0 \\
VAX & 12 & 0 & \\
\hline
\end{tabular}




\section{Software*}

Grophics

MacDraw II

MacPaint

(8)

(6)

Project Management MacProject II

Languages/Compilers

GW Basic

VAX Fortuan

VAX C

Utility

DOS
Spreadsheet

Multiplan

Lotus 1-2-3

(1)

(1)

Integraied Packages

Excel

(2)

Communication/Terminal Emulation

VTerm

(4)

Kermit

(6)

CAE/CAD
(1)

(1)

(1)

* The numbers of software packages are estimates.

\section{Network Connections}

\section{Existing \\ FY1991}

Lai-wide Ethernet

TCP/IP

DECnct

Both
22

9

10

3

\section{Estimated Total} FY1992

22

9

10

Planned activities and/or applications to be implemented during the next fiscal year in the areas of $(1)$ scientific work stations, (2) personal computing. (3) local area networking, and (4) office automation:

1. Scientific Work Stations: None planned.

2. Personal Computiı: None planned.

3. Local Area Networking: None planned

4. Office Automation: None planned. 


\section{HUMAN RESOURCES (COST CENTER 410)}

\section{Primary Contacts}

Contact Person Subject Phone E-Mail Address

$\begin{array}{lll}\text { Gcorge Lales } & \text { All } & \text { 2-2987 }\end{array}$

\section{Software}

Word Processing/Editing

Word

KEDIT

Word for Winduws (30)

Integrated Packages

Windows 3.0

$\begin{array}{ll}\text { Existing } & \text { Existing } \\ \text { FY1990 } & \text { FY1991 }\end{array}$

32

45

Estimated Total FY1992

0

1

47

1

Database

DBase III Plus

(6)

Communication/Terminal Emulation

Kermit

IBM PC3270

Telex

Spreadsheet
Lotus 1-2-3
(3)

Excel

(20)

Org. Plus

Cobra

Graphics

Harvard Graphics (1)

\section{Network Connections}

\section{Existing \\ FY1991}

Local 3Com

Epson Server
45

45
Estimated Total FY1992

47

47 
Planned activities and/or applications to be implemented during the next fiscal year in the areas of $(1)$ scientific work stations. (2) personal computing. (3) local area networking, and (4) office automation:

1. Scientific Work Stations: None planned.

2. Personal Computing: Personal productivity tools. Graphics presentation pacicage.

3. Local Area Networking: Additional server; new operating system (Netwarc or LAN Man).

4. Office Automation: Electronic forms processing. Electronic mail. 


\section{INSPECTIONS (COST CENTER 336)}

\section{Primary Contacts}

Contact Person

Subject

Phone

E-Mail Address

Thomas Busse

Personal Computers

$2-5713$

N/A

\section{Personal Computers and Workstations}

Manufacturer

Existing

FY1990

IBM (PC, AT, XT, PS $/$ )
Existing

FY1991

3
Estimated Total FY1992

3

\section{Software}

Word Processing/Editing

Word

Database

DBase IV

(3)

Spreadsheet

Multiplar.

(3)

Planned activities and/or applications to be implemented during the nex fiscal year in the areas of (1) scientific work stations, (2) personal computing, (3) local area networking, and (4) office automation:

1. Scientific Work Stations: None planned.

2. Personal Computing: General officellab support with word processing, database, and spreadsheet programs continuing.

3. Local Area Networking: None planned.

4. Office Automation: None planned. 


\section{INTENSE PULSED NEUTRON SOURCE PROGRAM (COST CENTER 136)}

\section{Primary Contacts}

$\begin{array}{llll}\text { Contact Person } & \text { Subject } & \text { Phone } & \text { E-Mail Address } \\ \text { Ira Bresof } & \text { All } & 2-8705 & \text { BRESOF AT ANLPNS }\end{array}$

\section{Personal Computers and Workstations}

Manufacturer

IBM (PC, AT, XT, PS/2)

Apple (II, Macintosh)

Compaq

Existin

Existing
FY1991
11
19
7

\section{Estimated Total} FY1992

13

22

\section{Word Processors}

$\begin{array}{lccc}\text { Manufacturer } & \text { Existing } & \text { Existing } & \text { Estimated Total } \\ \text { FY1990 } & \text { FY1992 } \\ \text { IBM } & 0 & 0 & 0\end{array}$

\section{Software}

Word Processing/Editing

MacWrite

Word

Text Editor

Mass 11

WordPerfoct

Database

DBase III Plus (1)

Mass11-Manage (1)

DBascll Mac

integrated Packages

Excel

Utility

Misc. Macintosh

(59)

Graphics

$\begin{array}{ll}\text { MacDraw II } & (5) \\ \text { MacPaint } & (2) \\ \text { Mass11 Draw } & (1) \\ \text { KaleidaGraph } & (7) \\ \text { DeltaGraph } & \text { (1) }\end{array}$

Spreadsh

Multiplan (3)

Quattro

Communication/Terminal Emulation

Kermit

(8)

PCSA

(9)

CAE/CAD

Claris CAD

(1) 


\section{Network Connections}

Existing
FY1991
Fstimated Total
FY1992

Lab-wide Ethernet

DECnet

1

Lab-wide AppleTalk

2

Planned activities and/or applications to be implemented during the next fiscal year in the areas of (1) scientific work stations, (2) personal computing. (3) local area ne.working, and (4) office automation:

1. Scientific Work Stations: We expect to acquire two more scientific workstations for data acquisition/analysis in FY1992. We will continue to develop $x$-windows applications and evaluate UNIX RISC workstations.

2. Personal Computing: We expect to acquire five more personal computers in FY1992. We will experiment with $x$-windows applications on PCs.

3. Local Area Networking: We expect to add about three nodes to our LAN in FY1992.

4. Office Automation: We expect to select a new word processing software package in FY1992. 


\section{LODGING FACILITIES (COST CENTER 222)}

\section{Primary Contacts}

$\begin{array}{llll}\text { Contact Person } & \text { Subject } & \text { Phone } & \text { E-Mail Address } \\ \text { Pat Carson } & \text { All } & 2-2580 & \text { N/A }\end{array}$

\section{Personal Computers and Workstations}

$\begin{array}{lccc}\text { Manufacturer } & \text { Existing } & \text { Existing } & \text { Estimated Total } \\ \text { FY1990 } & \text { FY1992 } \\ \text { Mitac (PC) } & 2 & 2 & 3\end{array}$

\section{Software}

Word Processing/Editing

Word

Multiplan

Lotus 1-2-3

Spreadsheat

\section{Database}

DBase IV
(1)

Planned activities and/or applications to be implemented during the next fiscal year in the areas of (1) scientific work stations, (2) personal computing. (3) local area networking, and (4) office automation:

1. Scientific Work Stations: None planned.

2. Personal Computing: Purchase hardware for an additional workstation.

3. Local Aroa Networking: None planned.

4. Office Automation: Procurement of a 3 workstationed Property Management System from the market, with the capability of interfacing with the existing Astra Innroads Call accounting system. 
MATERIALS SCIENCE DIVISION (COST CENTER 105)

\begin{tabular}{|llcl|}
\hline & \multicolumn{3}{c|}{ Primary Contacts } \\
Contact Person & Subject & Phone & E-Mail Address \\
Vcrnon Pahnke & All & $2-4937$ & $\begin{array}{l}\text { nick pahnke@msdgatc223.msd.anl. } \\
\text { gov }\end{array}$ \\
\hline
\end{tabular}

\section{Personal Computers and Workstations}

Manufacturer

IBM (PC, AT, XT, PS/2)

Applc (II, Macintosh)

Compaq

Zenith

NEC

SUN

Silicon Graphics

VAX

Next

\section{Existing \\ FY1990}

19

116

5

4

2

0

0

0

0

\section{Existing \\ FY1991}

19
125
8
0
0
3
2
6
5

Estimated Total FY1992

19

125
8

0

0

3

2

6

\section{Word Processors}

$\begin{array}{lccc}\text { Manufacturer } & \text { Existing } & \text { Existing } & \text { Estimated Total } \\ \text { FY1990 } & \text { FYl99l } & \text { FY } 1992 \\ \text { IBM } & 3 & \text { N/A } & \text { N/A } \\ \text { NBI } & 3 & \text { N/A } & \text { N/A } \\ \text { Apple } & 10 & \text { N/A } & \text { N/A }\end{array}$

\section{Software}

Word Processing/Editing

MacWritc

Word

MacWritc II

Database

DBase III Plus

Filemaker II

Graphics

$$
\begin{aligned}
& \text { MacDraw II } \\
& \text { MacPaint } \\
& \text { Statevicw } \\
& \text { MacDraw } \\
& \text { MacDraft } \\
& \text { CricketGraph }
\end{aligned}
$$

Integrated Packages

Excel
(27)

(27) 


\section{Software (continued)}

Security

Nightwatch

(2)

Communication/Terminal Emulation

VersaTerm Pro

VersaTerm

(15)

(3)

Other
(1)

(1)

(47)
Languages/Compilers

LS Fortran

MacFortran

MPW Pascal

LS Pascal

LS C

(7)

Utility

Disk Express

SUM

(1)

\section{Network Connections}

Existing

FY1991

Lab-wide Ethernct

TCP/IP

DECnct

Lab-wide AppleTalk

Local AppleTalk

\begin{abstract}
Mathematica
\end{abstract}
(5)

(3)

(1)

Planned activities and/or applications to be implemented during the next fiscal year in the areas of (1) scientific work stations, (2) personal computing, (3) local area networking, and (4) office automation:

1. Scientific Work Stations: None planned.

2. Personal Computing: None planned.

3. Local Area Networking: Rewiring portion of MSD 223 Appletalk.

4. Office Automation: None planned. 


\section{MATERIALS AND COMPONENTS TECHNOLOGY (COST CENTER 114)}

\begin{tabular}{|llll|}
\hline & \multicolumn{3}{c|}{ Primary Contacts } \\
Contact Person & Subject & Phone & E-Mail Address \\
Bill Shack & Macintosh & $2-5137$ & wj_shack@mctgate.mct.anl.gov \\
& & & \\
\hline
\end{tabular}

\begin{tabular}{|lccc|}
\hline & Personal Computers and Workstations & \\
& Existing & Existing & Estimated To:al \\
Manufacturer & FY1990 & FY1991 & 37 \\
IBM (PC, AT, XT, PS/2) & 45 & 35 & 60 \\
Apple (II, Macintosh) & 66 & 55 & 2 \\
Compaq & 2 & 2 & 9 \\
Sun & 3 & 7 & 4 \\
Zcnith & 4 & 4 & 10 \\
PC Limited & 10 & 10 & 1 \\
AST Rescarch & 1 & 1 & 7 \\
Other & 7 & 7 & \\
\hline
\end{tabular}

\begin{tabular}{|lccc|}
\hline & Word Processors & & \\
& Existing & Existing & Estimated Tolal \\
Manufacturer & FY1990 & FY1991 & FY 1992 \\
Exxon/Vydcc & 1 & 1 & 1 \\
NBI & 2 & 2 & 2 \\
\hline
\end{tabular}




\section{Software*}

Word Processirg/Editing

MacWrite

Word

DBasc III Plus

Filemaker Pro

Integrated Packages

Excel

Languages/Compilers

$\begin{array}{lr}\text { Fortran } & (4) \\ \text { Pascal } & (4) \\ \text { Basic } & (30) \\ \text { C } & (5)\end{array}$

Utility

Diskfit
Super LascrSpool (25)
Graphics

MacDraw II

MacDraw

MacPaint

CricketDraw

CricketGraph

KaleidaGraph

Spreadsheet

Lotus

Security

SUM

Folder Lock

Disinfenctant

Communication/Terminal Emulation

VTerm

(15)

Kermit

QuickMail

(50)

Telnet

(30)

Mail Sender

CAE/CAD

MiniCad

(2)

Dreams

(1)

* The numbers of software packages are estimates.

\section{Network Connections}

Existing

FY1991

15 Nodes + 3 Gator Boxes

60 Nodes
Estimated Total FY1992

18 Nodes + 3 Gator Boxes 60 Nodes

(Fax Modem for E-mail Network) 
Planned activities andlor applications to be implemented during the next fiscal year in the areas of (1) scientific work stations, (2) personal computing. (3) local area networking, and (4) office auiomation:

1. Scientific Work Stations: Will add two more Sun Sparc 2 workstations.

2. Personal Computing: No major change's planned.

3. Local Area Networking: No major changes planned.

4. Office Automation: Will try to encourage staff to use E-MAlL/FAX rather than dedicated fax machines. 


\section{MATERIALS AND SERVICES DEPARTMENT (COST CENTER 315)}

\section{Primary Contacts}

$\begin{array}{llll}\text { Contact Person } & \text { Subject } & \text { Phone } & \text { E-Mail Address } \\ \text { Bobby Mark } & \text { Inventory } & 2-2933 & \text { N/A }\end{array}$

\section{Personal Computers and Workstat:ins}

Existing Existing Estimated Total

Manufacturer

FY1990

FY1991

FY1992

IBM (PC, AT, XT, PS/2)

24

24

24

Gatcway

0

\section{Software}

Word Processing/Editing

Word
KEDIT
Multimatc

Graphics

$$
\text { Chart }
$$

Dutabase

DBasc III Plus (7)

DBasc IV

FoxBasc 2.1

Integrated Packages

Excel

Spreadsheet

Multiplan

Communication/Terminal Emulation

$\begin{array}{lr}\text { Kermit } & (2) \\ \text { Relay Gold } & (12) \\ \text { Reflection } & (7) \\ \text { Crosstalk } & (1)\end{array}$

Utility

Norton Utilitics (1)

Other

Hazmat Doc
Sideways
Regscan 49
Report Writer
Word Link
Genifer
Xerox Formbase
Windows 3.0

(2) 
Planned activities and/or applications to be implemented during the next fiscal year in the areas of (I) scientific work stations. (2) personal computing. (3) local area networking, and (4) office automation:

I. Scientific Work Stations: None planned.

2. Personal Computing: Personal productivity tools utilized such as word processing, database,desktop publishing, and form and database generations.

3. Local Area Networking: None planned.

4. Office Automation: Bar coding. 


\section{MATHEMATICS AND COMPUTER SCIENCE (COST CENTER 145)}

\begin{tabular}{llrl|} 
& & Primary Contacts & \\
Contact Person & Subject & Phone & E-Mail Address \\
Genc Rackow & All & $2-7126$ & Rackow@mcs.anl.gov \\
\hline
\end{tabular}

\begin{tabular}{lccc} 
& Personal Computers & and & Workstations \\
& Exiriing & Exisiing & Estimated Total \\
Maumfacturer & FYI990 & FY1991 & FY1992 \\
IBM (PC AT, XT, PS/2) & 6 & 6 & 6 \\
Apple (I, Macintosh) & 1 & 1 & 1 \\
Sun & 58 & 58 & 70 \\
Zsnith & 2 & 2 & 2 \\
NcXT & 20 & 20 & 20 \\
\hline
\end{tabular}

\section{Word Processors}

$\begin{array}{lccc}\text { Manufacturer } & \text { Existing } & \text { Existing } & \text { Estimated Total } \\ \text { FYi990 } & \text { FY1991 } & \text { FY } 1992 \\ \text { NBI } & 0 & 0 & 0\end{array}$

\section{Software}

Word Processing/Editing

MacWrit?

(1)

Publisher $\quad(6)$

TcX

(6)

Languages/Compilers

LS C
GH Fortran
MSC

(1)

(1)

(2)

Database

DBase III Plus (1)

Eroff

Graphics

MacDraw II

(1)

(1)

Spreadsheet

Multiplan

(1)

Communication/Terminal Emulation vTerm

(6) 


\section{Network Connections}

$\begin{array}{lc}\text { Existing } & \text { Estimated Total } \\ \text { FY1991 } & \text { FY1992 }\end{array}$

Lab-wide Ethernet

TCP/IP

all machines

all machines

Planned activities andlor applications to be implemented during the next fiscal year in the areas of $(1)$ scientific work stations, (2) personal computing, (3) local area networking, and (4) office automation:

1. Scientific Work Stations: None planned.

2. Personal Computing: None planned.

3. Local Area Networking: None planned.

4. Office Automation: None planned. 


\section{MEDIA SERVICES DEPARTMENT (COST CENTER 260)}

\begin{tabular}{|lcrc|}
\hline & & Primary Contacts & \\
Contact Person & Subject & Phone & E-Mail Address \\
Loc Wagar & All & $2-5603$ & B20429 AT ANLVM \\
\hline
\end{tabular}

\section{Personal Computers and Workstations}

Manufacturer

IBM (PC, AT, XT, PS/2)

Apple (Macintosh)

Existing
FY1990
14
12

Existing FY1901

20
Estimated Total FY1992

$1420 \quad 2$

\section{Software}

Word Processing/Editing

WordPcrfoct (1 Mac, 6 PC)

Word

(11 Mac, $7 \mathrm{PC}$ )

Spreadsheet

Quaturo Pro

(6 PC)

Presentation Visuals

Persuasion

(4 Mac)

Dalabase

DBasc III Plus (11 PC)

FilcMaker Pro (1 Mac)

Communication/Terminal Emulation

Kermit

NCSA Telnet

(N/A Mac, PC)

(N/A Mac)
Graphics

$\begin{array}{ll}\text { MacDraw II } & \text { (3 Mac) } \\ \text { Canvas } & \text { (1 Mac) } \\ \text { Freehand } & \text { (1 Mac) } \\ \text { Claris CAD } & (1 \mathrm{Mac}) \\ \text { DeltaGraph } & (1 \mathrm{Mac}) \\ \text { KaleidaGraph } & (1 \mathrm{Mac}) \\ \text { Illustrator } & (3 \mathrm{Mac})\end{array}$

Deskıop Publishing

PageMaker (9 Mac)

QuarkXpress (1 Mac)

Utility

MacLink (1 Mac)

Norton Utilities (1 PC)

DOS Mounter (1 Mac)

Disinfectant (N/A Mac)

XTreePro Gold (7 PC)

VirusScan (N/A PC) 


\section{Network Connections}

Existing

FY1991

Lab-wide Ethernet

TCP/P Telnet

XNS (3Com)

LocalTalk (AppleShare,

QuickMail, AlisaTalk)

Local AppleTalk
5

15

12

12
Estimated Total

FY1992
10

20

15

Planned activities andlor applications to be implemented during the next fiscal year in the areas of (1) scientific work stations. (2) personal computing. (3) local area networking, and (4) office automation:

1. Scientific Work Siations: None planned.

2. Personal Computing: Acquire hardware and software to implement photo library reorganization project, including image scanning to optical disk.

3. Local Area Networking: Acquisition of a router to isolate our 3Com Server from the Site-wide Ethernet and restrict it to our local Ethernet.

4. Office Automation: Print to AppleTalk printers from PCs on local Ethernet. 


\section{MOTION PICTURE UNIT (COST CENTER 276)}

\begin{tabular}{|llll|}
\hline & \multicolumn{3}{c|}{ Primary Contacts } \\
Contact Person & Subject & Phone & E-Mail Address \\
Gail Farmcr & IBM & $2-7451$ & N/A \\
& & & \\
\hline
\end{tabular}

Personal Computers and Workstations

Manufacturer

IBM (PC, AT, XT, PS/2)

Amiga 2000

MacIntosh

Existing
FY1990
2
1
0

Existing
FY1991
2
1
2

Estimated Total FY1992

$\begin{array}{lll}2 & 2 & 2 \\ 1 & 1 & 1 \\ 0 & 2 & 2\end{array}$

\section{Software}

Word Processing

WordPerfect 5.1 (2)

WordPerfect Mac (1)

Word

(1)

Spreadsheet

Lotus 1-2-3

\section{Database}

DBase III Plus (1)

Filemaker

(1)

Other

$\begin{array}{ll}\text { DigiView } & (1) \\ \text { Provideo Plus } & (1) \\ \text { VirleaScape } & (1) \\ \text { Scuipt/Animate3D } & (1) \\ \text { Aegis Animator } & (1) \\ \text { Labels Unlimited } & (1)\end{array}$


Planned activities andlor applications to be implemented during the next fiscal year in the areas of (1) scientific work stations, (2) personal computing. (3) local area networking, and (4) office automation:

1. Scientific Work Stations: None planned.

2. Personal Computing: None planned.

3. Local Area Networking: None planned.

4. Office Automation: None planned. 


\section{NATIONAL ENERGY SOFTWARE CENTER (COST CENTER 246)}

\begin{tabular}{|llcl|}
\hline & \multicolumn{3}{c|}{ Primary Contacts } \\
Contact Person & Subject & Phone & E-Mail Address \\
& & & \\
Cathy Eybcrger & General & $2-7575$ & B21675 AT ANLNESC \\
Frank Degges & Networking & $2-7680$ & B28194 AT ANLNESC \\
Jan Carter & Inventory & $2-7250$ & B27681 AT ANLNESC \\
\hline
\end{tabular}

\section{Personal Computers and Workstations}

Manufacturer

IBM (PC, AT, XT, PS $/ 2)$

Compaq

Existing
FY1990
3
1

\section{Existing}

FY1991

3

\section{Estimated Total} FY1992

\section{Word Processors}

Manufacturer

\section{Existing}

FY1990

Existing

FY1991

Estimated Total

3

1

Wang

4

4

4

\section{Software}

Word Processing/Editing

KEDIT

WordPerfoct

Spreadsheet

Lotus 1-2-3

Graphics

Harvard Graphics (1)

Communication/Terminal Emulation

$\begin{array}{cc}\text { Communication/Terminal } & \text { Emula } \\ \text { Kcrmit } & (2) \\ \text { IBM PC3270 } & (1) \\ \text { Conncct } & (1) \\ \text { PC/VT } & (1)\end{array}$

Databases

DBase IV (2)

Paradox

(1)

Languages/Compilers

MS Fortran

Lahey Fortran

MS QuickBasic

Arity Prolog

Ryan MacFarland Fortran (1)

MS C

(1)

(1)

(1)

Utility

Sidekick Plus (1) 
Planned activities and/or applications to be implemented during the next fiscal year in the areas of (1) scientific work stations, (2) personal computing. (3) local area networking, and (4) office automation:

1. Scientific Work Stations: None planned.

2. Personal Computing: None planned.

3. Local Area Networking: None planned.

4. Office Automation: None planned. 
OFFICE OF THE CHIEF FINANCIAL OFFICER (COST CENTERS 400, 401, 402, AND 403)

Primary Contacts

Contact Person

Subject

Phone

E-Mail Address

Paul Vignola

All

$2-7589$

B38039 AT ANLVM

\section{Personal Computers and Workstations}

Manufacturer

\section{Existing}

FY1990

IBM (PC, AT, XT, PS/2)

Zenith Portable
42

3
Existing
FY1991
91
3

\section{Estimated Total} FY1992

100

\section{Software}

Word Processing/Editing

Multimatc

MS-Word for

Windows

Database
DBasc III Plus
DBase IV
$\mathrm{Q}+\mathrm{E}$

Spreadsheet

SuperCalc

Lotus 1-2-3

Excel

Quattro 2.0

Integrated Packages

Symphony

Communication/Terminal Emulation

\section{Kcrmit}

(2)

IBM PC 3270

Maxcss by $3 \mathrm{Com}$

(1)

Other

RODEPC

Disc 1099PC
Graphics

SuperImage
MS-Power Point

(2)

(1)

Deskıop Publishing

Byline

Project Management

SuperProject Expert

(2)

Languages/Compilers

4GL, Jr.

Metaview

MS Visual Basic

Utility

Norton Utilities

(1)

Sidekick

Operating Systems/Environments MS DOS

MS Windows 3.0 


\section{Network Connections}

$\begin{array}{lc}\text { Existing } & \text { Total } \\ \text { FY1991 } & \text { FY1992 }\end{array}$

Local 3Com

70

80

Planned activities and/or applications to be implemented during the next fiscal year in the areas of $(1)$ scientific work stations, (2) personal computing, (3) local area networking, and (4) office automation:

1. Scientific Work Stations: None planned.

2. Personal Computing: We will migrate from SuperCalc5 and Multimate spreadsheet and word processing packages to Microsoft's Excel and Word for Windows.

3. Local Area Networking: 3Com has abastoned its local area network platform. Two candidates exist as a replacemnt network operating system: MS LANManager and Novell Netware. We will have to migrate to one of these in the next two years.

4. Office Automation: None planned. 


\section{OFFICE OF THE DIRECTOR (COST CENTER 201)}

\section{Primary Contacts}

\begin{tabular}{|c|c|c|c|}
\hline Contact Person & Subject & Phone & E-Mail Address \\
\hline $\begin{array}{l}\text { Dennis Tussing } \\
\text { Pat Traub }\end{array}$ & $\begin{array}{l}\text { Internal Audit Office } \\
\text { Strategic Planning Office } \\
\text { Office of the Director }\end{array}$ & $\begin{array}{l}2-3035 \\
2-4253\end{array}$ & $\begin{array}{l}\text { B35139 AT } \\
\text { PKT AT Al }\end{array}$ \\
\hline
\end{tabular}

\section{Personal Computers and Workstations}

Manufacturer

IBM (PC, AT, XT, PS/2)

Gatcway 2000
Existing

FY1990

10

0
Existing

FY1991

7

6
Estimated Total

FY1992

7

6

\section{Word Processors}

Manufacturer

Existing

FY1990

Existing

FY1991

0

stimated Total

FY 1992

0

NBI

8

\section{Software}

Word Processing/Editing

Multimatc version 4

DBase IV

Word Perfoct

Powerpoint for Windows

Grammatic IV
(5)

(3)

(7)

(3)

(3)

Desktop Publishing

Always

Excel

Communication/Terminal Emulation

Kermit

(2)

Spreadsheet

$\begin{array}{ll}\text { Multiplan } & (1) \\ \text { Lotus 1-2-3 } & (3) \\ \text { Quattro } & \text { (1) }\end{array}$

Database

DBase III Plus

(3)

Languages/Compilers

Basic

Windows 386
(4)

(1) 


\section{Software (continued)}

Other

Flowchart IIt (1)

Sidckick (1)

Norton Adv. (1)

\section{Network Connections}

Existing

FY1991
Estimated Total

FY1992

Lab-wide Ethernet

TCP/IP

0

0

Planned activities and/or applications to be implemented during the next fiscal year in the areas of (1) scientific work stations, (2) personal computing, (3) local area networking, and (4) office automation:

1. Scientific Work Stations: None planned.

2. Personal Computing: None planned.

3. Local Area Networking: 3Com or Novell

4. Office Automation: None planned. 


\section{OFFICE OF PUBLIC AFFAIRS (COST CENTER 275)}

\begin{tabular}{llll|}
\hline & \multicolumn{3}{c|}{ Primary Contacts } \\
Contact Person & Subject & Phone & E-Mail Address \\
Marilyn Witukofski & All & $2-5579$ & WRM AT ANLADM1 \\
\hline
\end{tabular}

\begin{tabular}{|c|c|c|c|}
\hline \multicolumn{4}{|c|}{ Personal Computers and Workstations } \\
\hline Manufacturer & $\begin{array}{l}\text { Existing } \\
\text { FY1990 }\end{array}$ & $\begin{array}{l}\text { Existing } \\
\text { FY1991 }\end{array}$ & $\begin{array}{c}\text { Estimated Total } \\
\text { FY1992 }\end{array}$ \\
\hline $\begin{array}{l}\text { IBMi (PC, AT, XT, PS/2) } \\
\text { Apple (II, Macintosh) } \\
\text { Vydec } 1800\end{array}$ & $\begin{array}{l}1 \\
2 \\
1\end{array}$ & $\begin{array}{c}1 \\
15 \\
1\end{array}$ & $\begin{array}{c}1 \\
15 \\
1\end{array}$ \\
\hline \multicolumn{4}{|c|}{ Word Processors } \\
\hline Manufacturer & $\begin{array}{l}\text { Existing } \\
\text { FY1990 }\end{array}$ & $\begin{array}{l}\text { Existing } \\
\text { FY1991 }\end{array}$ & $\begin{array}{l}\text { Estimated Total } \\
\text { FY } 1992\end{array}$ \\
\hline NBI & 7 & 8 & 8 \\
\hline
\end{tabular}

\section{Software}

Word Processing/Editing

Microsoft Word

FilcMaker

HyperCard

MultiFinder

Graphics

MacDraw II

(10)

MacPaint

Deskıop Publishing

PagcMaker

(6)

Integrated Packages

Excel

(1)

Communication/Terminal Emulation

Kermit

(6)

\section{Network Connections}

\section{Existing \\ FY1991}

Estimated Total

FY1992 
Planned activities and/or applications to be implemented during the next fiscal year in the areas of (1) scientific work stations, (2) personal computing. (3) local area networking, and (4) office automation:

1. Scientific Work Stations: None planned.

2. Personal Computing: None planned.

3. Local Area Networking: None planned.

4. Office Automation: None planned. 


\section{PHYSICAL RESEARCH (COST CENTER 273)}

\begin{tabular}{llrll} 
& & & \\
& & Primary & Contacts & \\
Contact PersGi: & Subject & Phone & E-Mail Address \\
Emest VanBcrkum & All & $2-3750$ & B07412 AT ANLVM \\
\hline
\end{tabular}

\begin{tabular}{|cccc|}
\hline & Personal Computers and Workstations & \\
& Existing & Existing & Estimated Total \\
Manufacturer & FY1990 & FY1991 & FY1992 \\
Apple (II, Macintosh) & 3 & 3 & 4 \\
\hline
\end{tabular}

\begin{tabular}{|lccc|}
\hline & Word Processors & & \\
& Existing & Existing & Estimated Total \\
Manufacturer & FY1990 & FY1991 & FY 1992 \\
NBI & 1 & 0 & 0 \\
\hline
\end{tabular}

\section{Software}

Word Processin $\sigma_{6}$ Editing MacWrite (1)

Word (2)

)

Graphics

$\begin{array}{ll}\text { MacDraw II } & \text { (1) } \\ \text { MacPaint } & (1) \\ \text { SuperPaint } & (1) \\ \text { MacDraft } & \text { (1) }\end{array}$

(1)

(1)
Desktop Publishing

More

(1)

\section{Network Connections}

Existing

FY1991

Local AppleTalk
3

\section{Estimated Total FY1992}


Planned activities and/or applications to be implemented during the next fiscal year in the areas of (1) scientific work stations, (2) personal computing. (3) local area networking, and (4) office automation:

1. Scientific Work Stations: None planned.

2. Personal Computing: Continue use of PCs for word processing, spreadsheets, graphics, and electronic mail.

3. Local Area Networking: None planned.

4. Office Automation: None planned. 


\section{PLANT FACILITIES AND SERVICES (COST CENTER 506)}

\section{Primary Contacts}

Contact Person

Subject

Phone

E-Mail Address

Duanc Bradlcy

All

$2-7307$

B15692A AT ANLVM

\section{Personal Computers and Workstations}

Manufacturer

$$
\begin{aligned}
& \text { Existing } \\
& \text { FY1990 }
\end{aligned}
$$

IBM (PC, AT, XT, PS/2)

AT\&T

Gateway 2000

Amcrican Mituc

Epson

$\begin{array}{ll}7 & 7 \\ 2 & 2 \\ 0 & 2 \\ 1 & 1 \\ 1 & 1\end{array}$

Estimated Total FY1992

Existing
FY1991
7
2
2
1
1

\section{Word Processors}

Manufacturer

Existing

FY1990

NBI

4
Existing

FY1991

5
Estimated Total FY 1992

\section{Software}

Dalabase

DBase IV

SupcrCalc IV

(3)

(1)

Communication/Terminal Emulation

Procomm

Kermit

(1)

(1)
Spreadsheet

Multiplan

(1)

Word Processing

WordPerfect (1)

NBI 
Planned activities and/or applications to be implemented during the next fiscal year in the areas of (1) scientific work stations, (2) personal computing, (3) local area networking, and (4) office automation:

1. Scientific Work Stations: None planned.

2. Personal Computing: May replace $\mathrm{NBI}$ equipment.

3. Local Area Networking: None planned.

4. Office Automation: None planned.

$-118-$ 


\section{PROCUREMENT DEPARTMENT (COST CENTER 322)}

\begin{tabular}{|llcl|}
\hline & & Primary Contacts & \\
Contact Person & Subject & Phone & E-Mail Address \\
Norman Goct\% & All & $2-7028$ & N/A \\
& & & \\
\hline
\end{tabular}

\begin{tabular}{lccc|} 
& Personal Computers and Workstations & \\
& Existing & Existing & Estimated Total \\
Manufacturer & FY1990 & FY1991 & FY1992 \\
IBM & 31 & 39 & 39 \\
Apple & 0 & 1 & 1 \\
Gatcway & 0 & 1 & 1 \\
\hline
\end{tabular}

\section{Word Processors}

$\begin{array}{lccc} & \text { Existing } & \text { Existing } & \text { Estimated Total } \\ \text { Manufacturer } & \text { FY1990 } & \text { FY1991 } & \text { FY } 1992 \\ \text { IBM } & 3 & 0 & 0 \\ \text { Lanicr } & 0 & 0 & 0\end{array}$

\section{Software}

Word Processing/Editing

Word

Graphics SuperChart

SuperImage

(1)

(1)

Database

DBase III Plus

(3)

Spreadsheet

Multiplan

Spreadsheet

Communication/Terminal Emulation

Reflections

(11)

Utility

Windows
Excel

(3) 


\section{Network Connections}

Existing
FYlimated Tolal
FYl

Local 3Com

1

1

Planned activities and/or applications to be implemented during the next fiscal year in the areas of $(1)$ scientific work stations, (2) personal computing, (3) local area networking, and (4) office automation:

1. Scientific Work Stations: None planned.

2. Personal Computing: Personal Productivity tools will be used, such as word processing, database, and 2nd spreadsheet to facilitate administrative and managerial functions.

3. Local Area Networking: Plan to replace current network hardware due to continued failure and to meet the needs of current procurement databases being rewritten/updated. Currently, research is being done on future network operating system upgrade from 3 Com $3+$ Share.

4. Office Automation: Current local area network houses office productivity application software (databases. word processors) allowing the sharing of peripherals such as hard drive and printers. 


\section{REACTOR ANALYSIS (COST CENTER 116)}

\section{Primary Contacts}

Contact Person

Subject

Phone

E-Mail Address

Kim Lalumendre

Inventory

$2-7720$

N/A

\section{Pirsonal Computers and Workstations}

Manufacturer

IBM (PC, AT, XT, PS/2)

Applc (II, Macintosh)

Compaq

Microstar

Digital

Gatcway

Existing
FY1990
14
1
1
3
1
9

\section{Existing FY1991}

11
1
1
3
1
0

\section{Estimated Total FY1992}

\section{Word Processors}

$\begin{array}{lccc}\text { Manufacturer } & \text { Existing } & \text { Existing } & \text { Estimated Total } \\ \text { FY1990 } & & \text { FY1991 } & \text { FY } 1992 \\ \mathrm{NBI} & 0 & 0 & 0\end{array}$

\section{Software}

Word Processing/Editing

WordPerfect

(10)

Spreadsheet

MacWritc

(2)

Lotus 1-2-3

(3)

Project Manugement

AEC Information Manager

(1)

Languages/Compilers

$\begin{array}{ll}\text { Basic } & (4) \\ \text { Fortran } & (3) \\ \text { Pascal } & (2)\end{array}$

Utility

Symantcc

(2) 


\section{Network Connections}

Existing
Estimated Total
FYl992

Local ApplcTalk TOPS

Planis: tactivities and/or applications to be implemented during the next fiscal year in the areas of $(1)$ scientific work stations, (2) personal computing, (3) local area networking, and (4) office automation:

1. Scientific Work Stations: None planned.

2. Personal Computing: None planned.

3. Local Area Networking: None planned.

4. Office Automation: None planned. 


\section{REACTOR ENGINEERING (COST CENTER 112)}

\begin{tabular}{|llcl|}
\hline & \multicolumn{3}{c|}{ Primary Contacts } \\
Contact Person & Subject & Phone & E-Mail Address \\
Jcriy Gaston & Inventory & $2-4563$ & N/A \\
& & & \\
\hline
\end{tabular}

\begin{tabular}{|lccc|}
\hline & Personal Computers & and & \\
& Existing & Existing & Estimated Total \\
& FY1990 & FY1991 & FY1992 \\
Manufacturer & 23 & 21 & 23 \\
IBM (PC, AT, XT, PS/2) & 2 & 15 & 17 \\
Apple (II, Macintosh) & 4 & 9 & 9 \\
Compaq & 3 & 3 & 3 \\
Sun & 6 & 2 & 2 \\
AST Rescarch & 1 & 0 & 0 \\
Kaypro & 9 & 6 & 6 \\
Lcading Edgc & 1 & 32 & 41 \\
Standard Brands & 2 & 1 & 1 \\
Hcwlcu Packard & & & \\
\hline
\end{tabular}

\section{Word Processors}

$\begin{array}{lccc} & \text { Existing } & \text { Existing } & \text { Estimated Total } \\ \text { Manufacturer } & \text { FY1990 } & \text { FYI991 } & \text { FY } 1992 \\ \text { NBI } & 10 & 0 & 0 \\ \text { Interpro } & 1 & 0 & 0\end{array}$

\section{Software}

Word Processing/Editing

Word

WordPerfoct

WordStar

(5)

(28)

(2)

Database

DBase III Plus

Project Management

MicroPlanner

Timclinc
(2)
Graphics

Cricket Graph

(2)

Cricket Draw

(2)

Spreadsheet

Lotus 1-2-3

Languages/Compilers

Pascal

Fortran

Basic 


\section{Software (continued)}

\section{Utility}

$\begin{array}{ll}\text { Norton Utilitics } & \text { (8) } \\ \text { CAD } & \text { (1) }\end{array}$

CAE/CAD

\section{Network Connections}

Existing

FY1991

Lab-wide Ethernet

TCP/IP

DECnet
42

1

\section{Estimated Total \\ FY1992}

50

1

Planned activities andlor applications to be implemented during the next fiscal year in the areas of (1) scienific work stations, (2) personal computing, (3) local area networking, and (4) office automation:

1. Scientific Work Stations: None planned.

2. Personal Computing: None planned.

3. Local Area Networking: None planned.

4. Office Automation: None planned. 


\section{SECRETARIAL AND CLERICAL SERVICES (COST CENTER 416)}

Sec Human Resources (Cost Center 410). Secretarial and Clerical Services and Human Resources share the same word processors, personal computers, workstations, and associated software; however, for budget purposes the cost centers are listed individually. 


\section{SECURITY (COST CENTER 232)}

\section{Primary Contacts}

Contact Person

Rich Ramos
Subject

Inventory
Phone

$2-5739$
E-Mail Address

N/A

\section{Personal Computers and Workstations}

$\begin{array}{lccc}\text { Manufacturer } & \begin{array}{c}\text { Existing } \\ \text { FY1990 }\end{array} & \text { Existing } & \text { Estimated Tor } \\ \text { FY1992 } \\ \text { IBM } & 5 & 12 & 13 \\ \text { AT\&T } & 2 & 2 & 2 \\ \text { Mitac } & 6 & 6 & 6 \\ \text { Galcway } & 0 & 2 & 2 \\ \text { Kinspao } & 0 & 1 & 1\end{array}$

\section{Software}

Word Processing/Editing

Multimatc Advantage II

WordPcrfoct

(1)

Relay Gold

ProCom
Database

DBase IV

(6)

DBase III Plus

FoxPro

(1)

(1)

Graphics

Harvard Graphics (3)

Other
13

6

1

\section{Network Connections}

Existing

FY1991
Estimated Total FY1992 
Planned activities andlor applications to be implemented during the next fiscal year in the areas of $(1)$ scientific work stations, (2) personal computing, (3) local area networking, and (4) office automation:

1. Scientific Work Stations: None planned.

2. Personal Computing: None planned.

3. Local Area Networking: Continued use of existing to network Safeguards and Security.

1. Office Automation: None planned. 


\section{SPECIAL MATERIALS (COST CENTER 150)}

\section{Primary Contacts}

Contact Person

Subject

Phone

E-Mail Address

Shirlcy Stogsdill

Workstations

$2-6774$

N/A

\section{Personal Computers and Workstations}

Manufacturer

IBM (PC, AT, XT, PS/2)

PC Limited

Dell

AT\&T

AMQ

Transdata

\section{Existing \\ FY1990}

2

1

3

1

1

\section{Existing \\ FY1991}

Estimated Total FY1992

\section{Software}

Word Processing/Editing

Multimate

WordPcrfoct

Multimatc LAN

Database

DBase III Plus

DBasc IV

(1)

(1)

Utility

Harvard Graphics (1)

Excel

Other

$\begin{array}{ll}\text { MS DOS } & \text { (6) } \\ \text { Star Exchange } & \text { (1) } \\ \text { PC DOS } & \text { (1) }\end{array}$

\section{Network Connections}

Existing

FY1991

Local 3Com
1

\section{Estimated Total} FY1992 
Planned activities and/or applications to be implemented during the next fiscal year in the areas of (I) scientific work stations. (2) personal computing, (3) local area networking, and (4) office awsmation:

1. Scienufic Work Stations: None planned.

2. Personal Computing: None planned.

3. Local Area Networking: None planned.

4. Office Aut mation: None planned. 


\section{SPECIAL PROJECTS OFFICE (COST CENTER 197)}

\section{Primary Contacts}

Contact Person

Subject

Phone

E-Mail Address

Debra Petersen

All

$2-7561$

N/A

Personal Computers and Workstations

Manufacturer

IBM (PC, AT, XT, PS/2)

Compaq

Zenith

Delta Data

Compaq LTE 286

Apple (Macintosh)

\section{Existing \\ FY1990}

24

28

2

2

0

\section{Existing \\ FY1991}

24

38

1

2

1
Estimated Total FY1992

\section{Software}

Word Processing/Editing

WordPerfect

WordPerfect LAN (4)

WordPerfect

Russian

Bilingual Solutions (3)

Microsoft Word (1)

Grammatik IV

(4)

Graphics

$\begin{array}{ll}\text { GemDraw } & (16) \\ \text { Other Gem } & (16) \\ \text { DrawPerfoct } & \text { (2) }\end{array}$

Desktop Publishing

Ventura

Pagemaker

(1)

DBase IV

DataPerfoct

Clipper

PC Album

Spreadsheet

Lotus 1-2-3

Symphony

Project Management

SuperProject

(4)

Integrated Packages

Excel

(6)

Framework

Communication/Terminal Emulation

$\begin{array}{ll}\text { Kermit } & (4) \\ \text { Bitcom } & (7) \\ \text { Carbon Copy } & \text { (2) }\end{array}$


Software (continued)

Utility

Mace

(4)

Other

Think C

WP Library

Harvard Project
(1)

(4)

(1)

\section{Network Connections}

$\begin{array}{lc}\text { Existing } & \text { Estimated Total } \\ \text { FY1991 } & \text { FY1992 }\end{array}$

Lab-wide Ethernct

TCP/IP

Local 3Com

(2)

(4)
AutoCAD
EasyCAD

Planned activities and/or appiications to be implemented during the next fiscal year in the areas of (I) scientific work stations. (2) personal corr'juting. (3) local area networking, and (4) office automation:

1. Scientific Work Stations: None planned.

2. Personal Computing: None planned.

3. Local Area Networking: None planned.

4. Office Automation: Plan to automate travel, training schedules, procurements, and other accounting functions using PCs. 


\section{SUPPORT SERVICES DIVISION (COST CENTER 216)}

\begin{tabular}{|llll|}
\hline & & Primary Contacts & \\
Contact Person & Subject & Phone & E-Mail Address \\
Virginia Biondi & All & $2-6182$ & N/A \\
\hline
\end{tabular}

\section{Personal Computers and Workstations}

Manufacturer

IBM (PC, AT, XT, PS/2)

Compaq

MacIntosh IICX

Gateway 2000 486/33

Existing
FY1990
7
1
0
0

Existing
FY1991
8
1
1
1

\section{Estimated Total FY1992}

8

1

1

\section{Word Processors}

$\begin{array}{cccc} & \text { Existing } & \text { Existing } & \text { Estimated Total } \\ \text { Manufacturer } & \text { FY1990 } & \text { FY1991 } & \text { FY } 1992\end{array}$

NBI

1

\section{Software}

Word Processing/Editing

Word

WordPcrfect

Mac Word

(1)

Database

DBase III Plus

DBase IV

Foxbase 2.0

Project Management

TimeLine
Graphics

Gem Draw

SuperChart

SuperImage

(1)

Microsoft Char

(1)

(2)

Spreadsheet

Multiplan

Quattro

Lotus 1-2-3

Mac Excel

(3)

(3)

(1)

(1)

Languages/Compilers

Clipper
(1) 


\section{Software (continued)}

Communication/Terminal Emulation Utility

Kermit (8)

Relay Gold (1)

Reflections (2)

Norton Utilities (1)

PC Tools

Other
ReportWriter
Genifer
(1)
Company Ladder (1)
dAnalyst Goid
(1)

\section{Network Connections}

Existing

FY1991

Local 3Com

Novell Nelware V.3.11
1
Estimated Total

FY1992

0

Planned activities and/or applications to be implemented during the next fiscal year in the areas of (1) scientific work stations, (2) personal computing, (3) local area networking, and (4) office automation:

1. Scientific Work Stations: None planned.

2. Personal Computing: Continued PC connection to mainframe via remote dial-in (modems); personal productivity tools utilized such as wordprocessing, databases and utilities, 2 nd spreadsheets aiding in administrative and managerial functions.

3. Local Area Networking: Upgraded existing 3Com 3+Share operating system to Novell Netware V 3.11. Replacement of older hardware as needed (3 server 3 file server).

4. Office Automation: Continued office productivity application software utilized (databases, spreadsheets, and word processors) and current existence of a local area network sharing hard drive and printers. 
TECHNICAL COMMUNICATION SERVICES DEPARTMENT (COST CENTER 265)

Primary Contacts

Contact Person

John Bocbinger

Jackie Griffith

Linda Halcy

\section{Subject}

Local Talk Network

PC Network

PCs/Macs

Word Processing

Graphics
Phone

$2-6997$

$2-3795$

$2-8820$
E-Mail Addres"

N/A

JJD AT ANLEESI

N/A

\section{Personal Computers and Workstations}

$\begin{array}{lccc} & \text { Existing } & \text { Existing } & \text { Estimated } \\ \text { Manufacturer } & \text { FY1990 } & \text { FY1991 } & \\ \text { IBM (PS/2) } & 0 & 5 & 5 \\ \text { Apple (II, Maclntosh) } & 0 & 12 & 3 \\ \text { Applc Portable Mac } & 0 & 2 & 2 \\ \text { Gatcway 2000 } & 0 & 19 & 10 \\ \text { Portable PCs } & 0 & 3 & 3 \\ \text { NBI PC } & 0 & 3 & 0\end{array}$

Word Processors and Network Servers

Manufacturer

NBI WP/WS

NBI WP/Server

NBI Unix Server

PC Network Scrver

\section{Existing \\ FY1990}

1
1
1
0

Existing
FY1991

1

1

1
Estimated Total FY 1992 


\section{Software}

Word Processing/Editing

Word Perfect (DOS) (25)

Word Perfect (Mac) (5)

MS Word (Mac)

MS Word (DOS)

Expressionist

Macwrite

(4)

(1)

\section{Communications}

Xtalk

MacLink Plus

Software Bridge

Whitc Knight

Graphics

Cricket Graph (3)

Adobc Collector's (2)

Aldus Pagemaker

Color

Certificatc Maker (1)

Frcedom of Press (2)

MacFlow

MapMaker

SupcrPaint

Visual Business Gra. (1)

Adobe Illustrator (1)

Aldus Pagemaker (4)

Ccrificate Library (1)

Graphics PainuI (1)

MacDraft

Math Typc

Vudco Work Acc. (1)
Database

File Maker Pro (2)

Excel

DbaseIII

(5)

Data Perfect

Plan Perfect

(1)

Fast Track Sch. (1)

Utilities

$\begin{array}{lll}\text { Norton (PC) } & \text { (1) } & \text { 386MAX } \\ \text { Norton (Mac) } & \text { (1) } & \text { QEMM } \\ \text { After Dark } & \text { (11) } & \text { SuitCase II } \\ \text { MacroMind Dir } & \text { (1) } & \text { LifeGuard } \\ \text { MicroMind Acc. (1) } & \text { Sticky Business } \\ \text { Sound Clips 1\&2 (1) } & \text { Timbuktu } \\ \text { Top Honors } & \text { (1) } & \text { Thunder } 7\end{array}$

(16)

(6)

(1)

(1)

(1)

(1)

(1)

Power Point

(5)

Adobe Type

Manager

Canvas

(4)

Cricket Presents (1)

Image Studio

(1)

Laser Award Maker (1)

Omni Page

(1)

Video WorksII

(1)

Versa Term Pro (1)

Adobe Streamline (1)

Aldus Persuasion (4)

Clip Art Library (1)

Kaleidagraph (2)

Map Art-Pict (1)

Pixel Paint Pro (1)

Visual Bus.\#5
Legend

Aldus

Frechand

Super 3D

DeltaGraph

MacDraw II

Map Art-Paint

RetroSpect

Draw Perfect

Adpbe Plus Pack

(5)

Adobe Type Libr (1)

The Curator (1)

Dreams

MacAllas Pro

Map art-EPS

Video Paint 


\section{Network Connections}

$\begin{array}{cc}\text { Existing } & \text { Estimated Total } \\ \text { FY1991 } & \text { FY1992 } \\ & \\ 26 & 36 \\ 13 & 18\end{array}$

Planned activities andlor applications to be implemented during the next fiscal year in the areas of (1) scientific work stations, (2) personal computing, (3) local area networking, and (4) office automation:

1. Scientific Work Stations: None planned.

2. Personal Computing: Continued growth and development of existing applications.

3. Local Area Networking: Change from current NBI network to either OS/2 Lan Man. Word Perfect Office, or Novell. Included with the implementation of a new PC Based network will be the addition of network servers. Exact amount cannot be determined until a final decision is made as to what network software will be used.

4. Office Automation: With the implementation of new network, both PCs and Mac will coexist, share resources, ani' have common mail capabilities. 
TECHNICAL INFORMATION SERVICES DEPARTMENT (COST CENTER 161)

\section{Primary Contacts}

Contact Person

Subject

Phone

E-Mail Address

Shannon Savage

All

$2-4221$

B27707TI AT ANLCV1

Personal Computers and Workstations

Manufacturer

IBM (PC, AT, XT, PS $/ 2$ )

Zenith

Terminals for AIM*

MAC IIC

\section{Existing}

FY1990

34
2
$16-20$
0

Existing
FY1991

46

2

31
Estimated Total FY1992

46

2

31

*Argonne Information Management System

\section{Software}

Word Processing/Editing

Word

KEDIT

Spreadsheet

Lotus 1-2-3

Communication/Terminal Emulation

Procomm +
Database

DBase III Plus

(3)

Languages/Compilers MS Cobol

(1)

Utility

Windows

Fastback + 
Planned activities and/or applications to be implemented during the next fiscal year in the areas of (1) scientific work stations, (2) personal computing, (3) local area networking, and (4) office automation:

1. Scientific Work Stations: Developing access through AIM to CD-ROM storage of scientific and technical information.

2. Personal Computing: Training and further use in Windows 3.0.

3. Local Area Networking: Install a 3-COM system and investigate hooking it into the Laboratorys' ethernet telecommunications system.

4. Office Automation: Investigate upgrading to Word for Windows. 


\section{TRAVEL OFFICE (COST CENTER 319)}

\begin{tabular}{|llcc|}
\hline & & Primary Contacts & \\
Contact Person & Subject & Phone & E-Mail Address \\
Lawrence McCurc & All & $2-4440$ & N/A \\
& & & \\
\hline
\end{tabular}

\section{Personal Computers and Workstations}

$\begin{array}{lccc}\text { Manufacturer } & \text { Existing } & \text { Existing } & \text { Estimated Total } \\ \text { FY1990 } & \text { FY1992 } \\ \text { IBM (PC, AT, XT, PS/2) } & 2 & 2 & 2\end{array}$

Software

Word Processing/Editing Word
Database

DBase III Plus

(2)

Planned activities andlor applications to be implemented during the next fiscal year in the areas of (1) scientific work stations. (2) personal computing. (3) local area networking, and (4) office automation:

1. Scientific Work Stations: None planned.

2. Personal Computing: None planned.

3. Local Area Networking: None planned.

4. Office Automation: None planned. 


\section{UTILITY SYSTEMS (COST CENTER 510)}

\begin{tabular}{|lccc|}
\hline & & Primary Contacts & \\
Contact Person & Subject & Phone & E-Mail Address \\
Jim Huggins & Manager & $2-5241$ & N/A \\
& & & \\
\hline
\end{tabular}

\section{Personal Computers and Workstations}

$\begin{array}{lccc}\text { Manufacturer } & \text { Existing } & \text { Existing } & \text { Estimated Total } \\ \text { FY1990 } & 1 & \text { FY1991 } & \text { FY1992 } \\ \text { IBM (PC, AT, XT, PS/2) } & 1 & 1 & 1\end{array}$

\section{Software}

Database
DBase IV
(1)

Planned activities and/or applications to be implemented during the next fiscal year in the areas of (I) scientific work stations, (2) personal computing. (3) local area networking, and (4) office automation:

1. Scientific Work Stations: None planned.

2. Personal Computing: None planned.

3. Local Area Networking: Plan to install a local area network to track maintenance deficiencies. Will replace application on IIP3000.

4. Office Automation: None planned. 


\section{VEHICLE MAINTENANCE (COST CENTER 316, 317, 503)}

\begin{tabular}{|llll|}
\hline & & Primary Contacts \\
Contact Person & Subject & Phone & E-Mail Address \\
Bctly Andrus & All & $2-7100$ & N/A \\
\hline
\end{tabular}

\begin{tabular}{|lccc|}
\hline \multicolumn{4}{c|}{ Personal Computers } \\
& Existing & Existing & \\
& FY1990 & FY1991 & Estimated Total \\
Manufacturer & 4 & 5 & FY1992 \\
IBM (PC, AT, XT, PS/2) & & 5 \\
\hline
\end{tabular}

\section{Software}

Word Processing/Editing

Word

Dalabase

WordPerfect (1)

LAN PACK (N/A)

Network Connections
Existing
FY1991

Local 3Com 
Planned activities and/or applications to be implemented during the next fiscal year in the areas of (1) scientific work stations, (2) personal computing, (3) local area networking, and (4) office automation:

1. Scientific Work Stations: None planned.

2. Personal Computing: Implementation of Word Perfect word processing.

3. Local Area Networking: Implementing of terminal at Grounds Department on 3Com Server. The application on this terminal for Grounds work is now operational..

4. Office Automation: Develop experience on Microsoft Word and Word Perfect word processing. 


\section{WASTE MANAGEMENT OPERATIONS (COST CENTER 505)}

\begin{tabular}{|llcl|}
\hline & \multicolumn{3}{c|}{ Primary Contacts } \\
Contact Person & Subject & Phone & E-Mail Address \\
Duane Bradlcy & All & $2-7307$ & B15692A AT ANLVM \\
\hline
\end{tabular}

\begin{tabular}{|lccc|}
\hline & Personai Computers and & \\
& & & \\
Manufacturer & Existing & Existing & Estimated Total \\
AT\&T & FY1990 & FY1991 & FY1992 \\
Epson & 1 & 2 & 2 \\
IBM & 0 & 9 & 9 \\
\hline
\end{tabular}

\section{Word Processors}

$\begin{array}{lccc} & \text { Existing } & \text { Existing } & \text { Estimated Total } \\ \text { Manufacturer } & \text { FY1990 } & \text { FY1991 } & \text { FY 1992 } \\ \text { NBI } & 1 & 0 & 0\end{array}$

\section{Software}

Spreadsheet

$\begin{array}{ll}\text { Multiplan } & \text { (1) } \\ \text { Lolus } & \text { (3) }\end{array}$

Graphics

Harvard Graphics

Communications/Terminal Emulation Procomm

(1)

Word Processing

NBI (1)

Word Perfect (3)

Microsoft Word (1)

Vatabases

DBase IV

(7) 
Planned activities and/or applications to be implemented during the next fiscal year in the areas of (1) scientifir work stations, (2) personal computing. (3) local area networking, and (4) office automation:

1. Scientific Work Stations: None planned.

2. Personal Computing: May replace NBI equipment.

3. Local Area Networking: None planned.

4. Office Automation: None planned. 

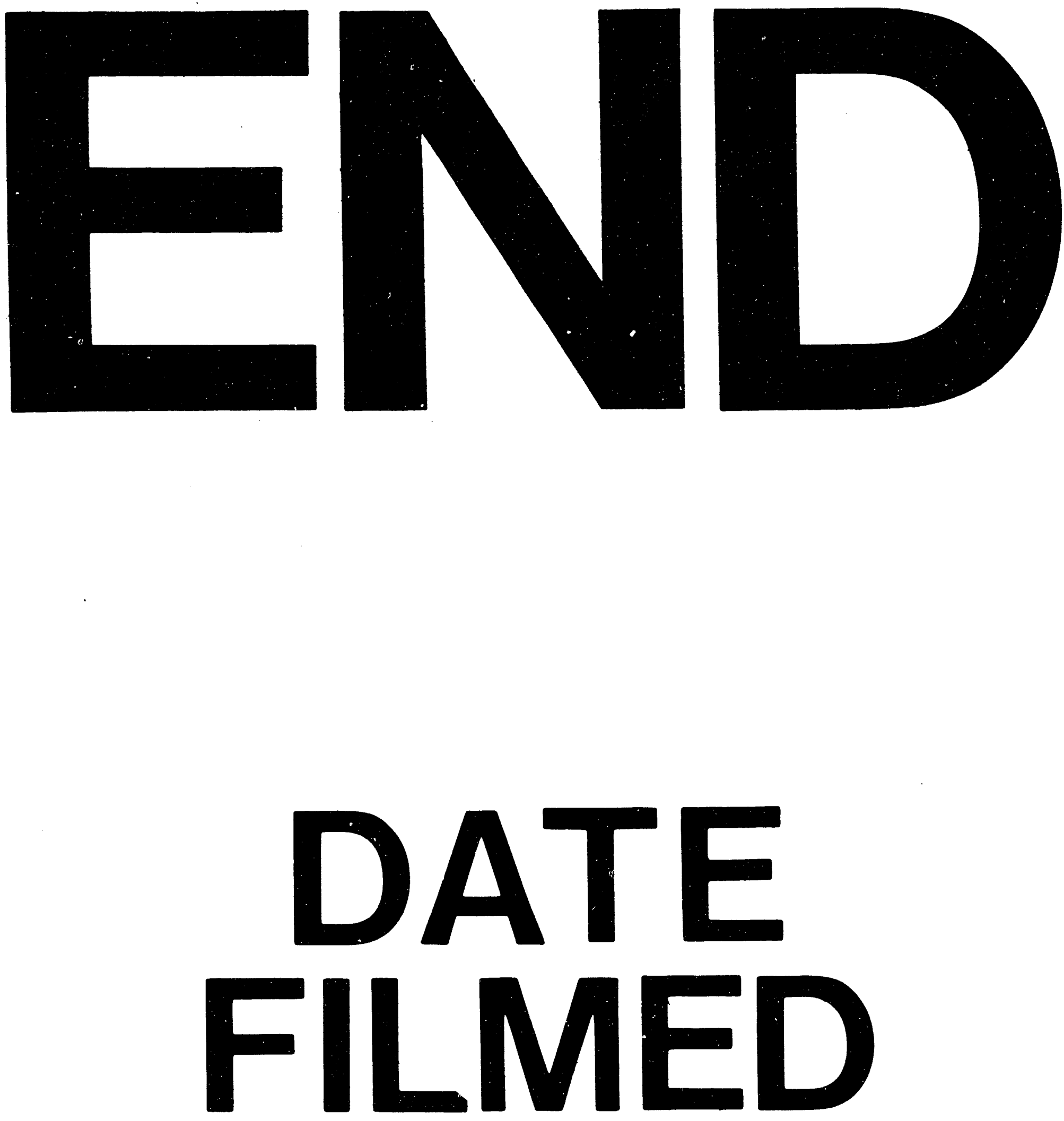

1

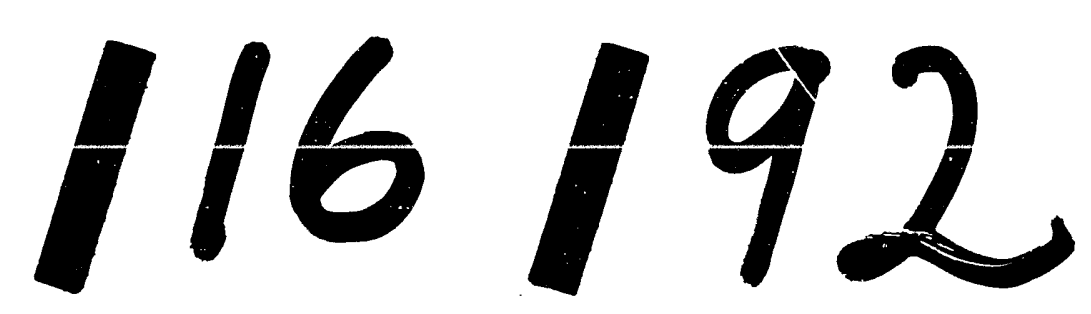


